\title{
A synoptic review and new infrageneric classification for the genus Haworthiopsis (Xanthorrhoeaceae: Asphodeloideae)
}

\author{
SEAN D. GILDENHUYS ${ }^{1, *} \&$ RONELL R. KLOPPER ${ }^{2} 3$ \\ ${ }^{1}$ Succulent Society of South Africa, P. O. Box 11017, Hatfield, 0028, South Africa; e-mail: gasteria@iburst.co.za \\ ${ }^{2}$ Biosystematics Research and Biodiversity Collections Division, South African National Biodiversity Institute, Private Bag X101, Preto- \\ ria,0001, South Africa; e-mail: r.klopper@sanbi.org.za \\ ${ }^{3}$ H.G.W.J. Schweickerdt Herbarium, Department of Plant Science, University of Pretoria, Pretoria, 0002, South Africa \\ *author for correspondence
}

\begin{abstract}
Haworthiopsis was established in 2013 to accommodate the species formerly classified under the subgenus Hexangulares of the genus Haworthia. This new genus is near-endemic to South Africa and found in most of the provinces of the country. It is also known from southern Namibia, Swaziland and possibly Mozambique. A total of 18 species are currently recognised in Haworthiopsis. However, this circumscription renders the genus paraphyletic with $H$. koelmaniorum sister to a polytomy comprising the rest of the Haworthiopsis taxa and Gasteria. In this contribution, seven new combinations are published (H. fasciata var. browniana, H. reinwardtii var. reinwardtii f. chalumnensis, H. reinwardtii var. reinwardtii f. kaffirdriftensis, $H$. reinwardtii var. reinwardtii f. olivacea, $H$. tessellata var. crausii, $H$. viscosa, $H$. scabra var. smitii) and two changes of status (H. koelmaniorum var. mcmurtryi, H. viscosa var. variabilis). An amplified description of the genus is provided. Six sections formerly recognised under Haworthia (subg. Hexangulares) are applied and adapted to Haworthiopsis, with some amendments. A new section, $H$. sect. Koelmaniorum, is described here. New combinations for hybrid taxa in Haworthiopsis that were previously described as species of Haworthia are given.
\end{abstract}

\section{Introduction}

Alooid and haworthioid species are included in the mainly southern African Xanthorrhoeaceae subfam. Asphodeloideae [following APG III (Angiosperm Phylogeny Group 2009)] or alternatively in Asphodelaceae: Alooideae [following Nyffeler \& Eggli (2010)]. In APG IV (Angiosperm Phylogeny Group 2016) the family circumscription is similar to that of APG III, but the family name is given as Asphodelaceae nom. cons. prop., according to the pending conservation proposal by Klopper et al. (2013). The genus Haworthiopsis Rowley (2013a: 4) comprises 18 species with a total of 25 recognised infraspecific taxa. It is centred in South Africa with most taxa being endemic or near-endemic to this country.

\section{Taxonomic history}

The taxonomic history of the species now placed in Haworthiopsis is quite complex. Bayer (1999) mentioned that the earliest written record of these Haworthia-like plants is contained in a list of aloes compiled in 1695 by the Germanborn South African physician and botanist, Hendrik (Heinrich) Bernard Oldenland, who was then superintendent of the Dutch East India Company's Garden in what is today Cape Town, South Africa. Of the 28 different "aloes" listed, four were haworthioid plants, and two of these were species now included in Haworthiopsis [H. venosa (Lamarck 1783: 89) Rowley (2013a: 4) and H. viscosa (Linnaeus 1753: 322) Gildenhuys \& Klopper comb. nov.]. Linnaeus (1753) similarly included all the alooid plants known to him at the time in Aloe Linnaeus (1753: 319), including these two species that are now placed in Haworthiopsis. Later, the French physician and botanist, Henri Auguste Duval, established the genus Haworthia Duval (1809: 7) to accommodate the group of Aloe species with smaller white bilabiate flowers. This genus was named in honour of the English naturalist, Adrian Hardy Haworth (1767-1833). 
Since the first written records of these plants, travel has become much easier, resulting in the discovery of numerous new species and populations of plants. With all these accumulated collections, it has become far more difficult to classify all the known units sensibly, and to supply names for species concepts that faithfully reflect the natural variation. Despite this, over the past 200 years many authors and enthusiasts have contributed to the knowledge, taxonomy and classification of the haworthioid plants. Unfortunately this has also resulted in a great deal of confusion, with long lists of synonyms and a tangle of often divergent concepts followed by various authors in different eras.

In recent years, scientific advances in molecular and phylogenetic research have provided new insights regarding the relationships of these plants (Grace et al. 2013; Daru et al. 2013), bringing about changes in the taxonomy of the alooid genera. Most of the species previously classified by Bayer (1976 to 1999; Bayer \& Manning 2012) under Haworthia subgen. Hexangulares (Uitewaal 1947a: 136) Bayer (1971:160) were put into the new genus Haworthiopsis. At the same time the genus Tulista Rafinesque (1840: 137) was resurrected to accommodate those of the former Haworthia subgen. Robustipedunculatae (Uitewaal 1947a: 136) Bayer (1971: 160). However, these taxonomic changes were marred by Rowley's concept of the genus Tulista, and errors in the relevant publication. Rowley (2013a \& b) combined disparate elements into his heterogeneous concept of Tulista, including not only the former subgenus Robustipedunculatae, but also the entire genus Astroloba Uitewaal (1947b: 53), as well as Aristaloe aristata (Haworth 1825: 280) Boatwr. \& J.C.Manning in Manning et al. (2014: 69) (then Aloe aristata Haw.), Poellnitzia rubriflora (Bolus 1920: 13) Uitewaal (1940a: 61) [Astroloba rubriflora sensu Manning \& Smith (2000: 53)], and three species from the former Haworthia subgen. Hexangulares [namely Haworthia koelmaniorum Obermeyer \& Hardy (1967: 1502), Haworthia pungens Bayer (1999: 188) and Haworthia viscosa (Linnaeus 1753: 322) Haworth (1812: 90)]. To add to the confusion, Haworthia viscosa was initially erroneously listed as both a species of Haworthiopsis and of Tulista (Rowley 2013a). Furthermore, Haworthia granulata Marloth (1910:39) was given two new combinations under the genus Haworthiopsis (Rowley 2013a). In a later amended version of this classification Rowley (2013b) corrected these mistakes by, amongst others, removing the combinations Haworthiopsis viscosa and Haworthiopsis venosa var. granulata (Marloth) Rowley (2013a: 5), and adding Haworthiopsis tessellata (Haworth 1824: 300) Rowley (2013b: 5) (see further comments under 'Taxonomic changes on species and infraspecific level in Haworthiopsis' below).

Later, Manning et al. (2014) made more coherent changes to the alooid genera. The concept of Tulista was adapted to only comprise the species of the former Haworthia subgen. Robustipedunculatae. Astroloba was reinstated as a separate and distinct genus. The species Tulista koelmaniorum (Oberm. \& Hardy) Rowley (2013a: 5-6) and Tulista pungens (M.B.Bayer) Rowley (2013a: 6) were also transferred to Haworthiopsis so that the genus correlated with the former Haworthia subgen. Hexangulares. Based on floral morphology the species of Haworthiopsis seem to be related, but questions remain regarding how closely related Haworthiopsis koelmaniorum (Oberm. \& Hardy) Boatwr. \& J.C.Manning in Manning et al. (2014: 70) might be to H. limifolia (Marloth 1910: 409) Rowley (2013a: 4) and H. tessellata, and how closely related these are to the more typical species of Haworthiopsis occurring much further to the south and southwest in South Africa.

To truly build a better understanding of these plants (especially at specifc and infraspecific level), an enormous amount of field work is still needed, for example as M. Bruce Bayer has done for Haworthia (in the broad sense) in recent decades, and as G.W. Reynolds did for Aloe. It is also true that every person's concepts of taxa vary, and the interpretation of their observations plays a vital role. In addition, much remains to be learned from in-depth molecular studies. Work done thus far on the phylogeny of the aloe family has resulted in greatly changed taxonomy in this group. The use of next generation DNA sequencing techniques should be investigated to ascertain its usefulness in resolving the remaining issues.

\section{Material and methods}

This paper provides a synopsis of Haworthiopsis with emphasis on the sectional classification of the genus. It is based on the study of Haworthiopsis plants in the field and in cultivation over many years, as well as an indepth analysis of literature relating to these taxa. Images of important type specimens were obtained from the JSTOR Global Plants repository [http://plants.jstor.org]. Herbarium acronyms follow Thiers (2015). 


\section{Distribution}

The genus Haworthiopsis is near-endemic to South Africa (see Fig. 1). All species are found only within South Africa, except for two that have their main distribution within South Africa, but are also present in neighbouring countries. The first and most widespread of these is H. tessellata, which is also found in southern Namibia. The second is H. limifolia, which is also present in Swaziland and possibly in southern Mozambique, though very little is known about its occurrence there.

Of the genera with Haworthia-like flowers, Haworthiopsis has the widest distribution in South Africa, occurring in eight of the nine provinces (see Table 1). This leaves only the Gauteng Province without any known records for the genus. However, the unique and very interesting relative Chortolirion Berger (1908: 72) [now included in Aloe sect. Chortolirion (A.Berger) Boatwr. \& J.C.Manning in Daru et al. (2012: 13)], with its Haworthiopsis-like flowers and Aloe kniphofioides-like bulbs and leaves, is found in this province.

TABLE 1. The provinces of South Africa, with the number of Haworthiopsis species and number of endemics represented in each province. A list of the relevant species occurring in each province is also provided. $(*=$ Species represented in more than one province)

\begin{tabular}{|c|c|c|}
\hline Province & Number of species (and endemics) & Species \\
\hline Eastern Cape & $\begin{array}{l}14 \text { Species } \\
\text { (10 endemic) }\end{array}$ & $\begin{array}{l}\text { H. attenuata } \\
\text { H. bruynsii } \\
\text { H. coarctata } \\
\text { H. fasciata } \\
\text { H. glauca } \\
\text { H. longiana } \\
\text { H. nigra* } \\
\text { H. pungens } \\
\text { H. reinwardtii } \\
\text { H. scabra* } \\
\text { H. sordida } \\
\text { H. tessellata* } \\
\text { H. viscosa* } \\
\text { H. woolleyi }\end{array}$ \\
\hline Western Cape & $\begin{array}{l}6 \text { Species } \\
(1 \text { endemic })\end{array}$ & $\begin{array}{l}\text { H. granulata* } \\
\text { H. nigra* } \\
\text { H. scabra* } \\
\text { H. tessellata* } \\
\text { H. viscosa* } \\
\text { H. venosa }\end{array}$ \\
\hline Northern Cape & 3 Species & $\begin{array}{l}\text { H. granulata* } \\
\text { H. nigra* } \\
\text { H. tessellata* }\end{array}$ \\
\hline Free State & 1 Species & H. tessellata* \\
\hline North-West & 1 Species & H. tessellata* \\
\hline Limpopo & 1 Species & H. koelmaniorum* \\
\hline Mpumalanga & 2 Species & $\begin{array}{l}\text { H. koelmaniorum* } \\
\text { H. limifolia* }\end{array}$ \\
\hline KwaZulu-Natal & 1 Species & H. limifolia* \\
\hline
\end{tabular}

\section{The sections of Haworthiopsis}

The former three subgenera of Haworthia s.l. [Haworthia, Hexangulares and Robustipedunculatae (as 'Robustipedunculares')] were established by Bayer (1971), deriving partly from the concept of Uitewaal (1947a). These subgenera have given rise to the three separate genera we now know as Haworthia s.str., Haworthiopsis and Tulista, respectively (Rowley 2013a; Manning et al. 2014). Below the genus level, sections have long been used in Haworthia s.l. by different authors, including Haworth (1821), Salm-Dyck (1836), Berger (1908), Smith (1950), Scott 


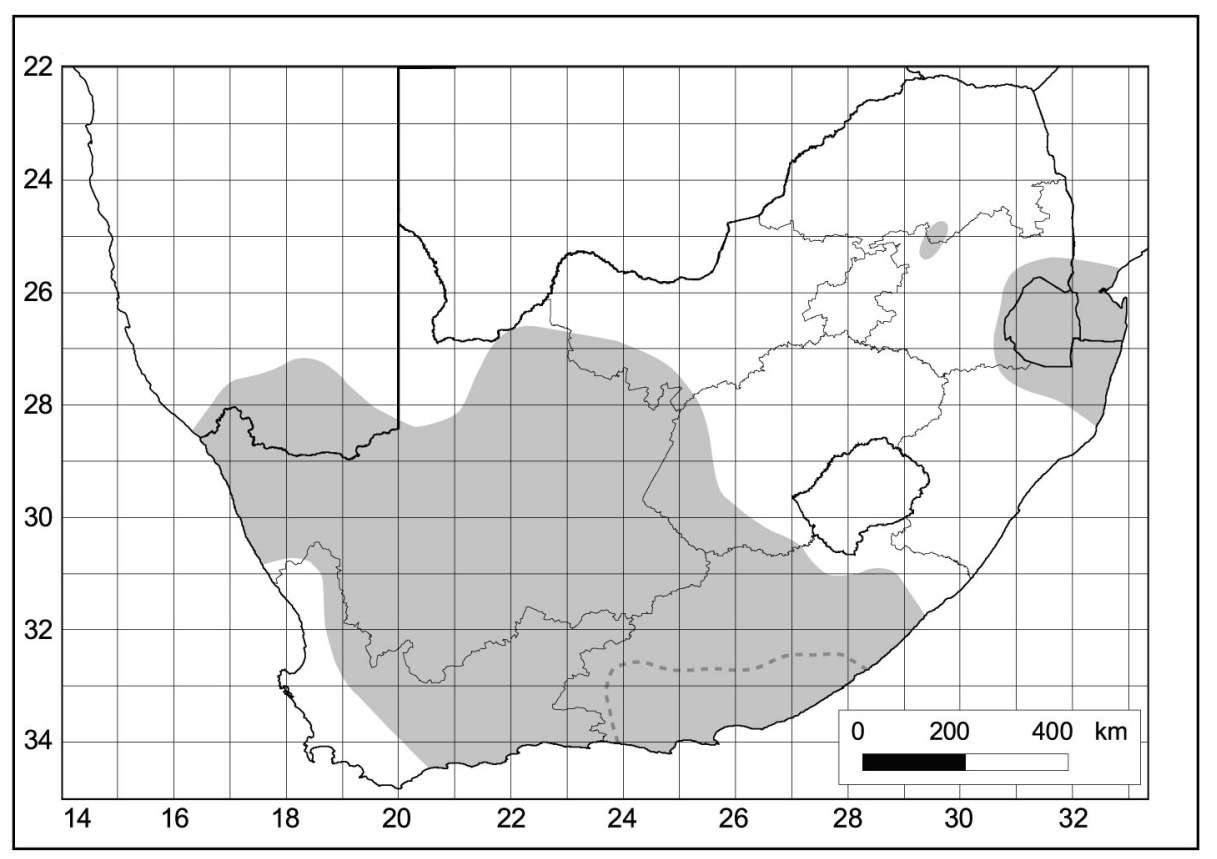

FIGURE 1. Potential distribution range of the genus Haworthiopsis (grey shaded area). The area demarkated in dashed lines contains the highest concentration of taxa.

(1985) and Breuer (2010). However, Bayer $(1976,1982,1999)$ did not make use of any of the previously established sections. To sensibly classify all the known taxa of Haworthia s.str. into sections is a formidable task, but within Haworthiopsis sections are easier to delimit, although the positioning of a few species is still uncertain.

According to a phylogram published by Manning et al. (2014: fig. 1) Haworthiopsis, as currently circumscribed, is not a monophyletic group (see Fig. 2 for a simplified version of this phylogram). Haworthiopsis koelmaniorum is recovered in a very well-supported clade (clade A in Fig. 2), that is sister to clades containing: 1) the main group of Haworthiopsis taxa together with Gasteria, and 2) Tulista, Aristaloe, Gonialoe and Astroloba (both sister clades lack bootstrap support). Rowley (2013a, 2013b) used the heterogeneous Tulista (grey shading in Fig. 2) to group all the seemingly outlying taxa (i.e. H. koelmaniorum, Tulista, Aristaloe, Gonialoe and Astroloba). In this sense, H. koelmaniorum was included in Tulista, but was later moved to Haworthiopsis by Manning et al. (2014). The broad concept of Tulista as implemented by Rowley (2013b) did not solve the problem of possible non-monophyly in Haworthiopsis sensu Rowley (2013b) (clade B in Fig. 2). This Haworthiopsis polytomy does contain some fairly well supported clades, but resolution is generally poor and no further taxonomic conclusions should be made based on these results. The main Haworthiopsis-Gasteria clade does not have strong bootstrap support. However, the Gasteria clade on its own, is well supported in all phylogenies published thus far (Treutlein et al. 2003, Manning et al. 2014) underlining the taxonomic integrity of this genus. The placement of especially $H$. koelmaniorum remains unsure and further in-depth phylogenetic studies on this and other Haworthiopsis taxa not currently included in molecular analyses need to be done to build a better understanding of their relationships. Before all Haworthiopsis taxa have been investigated and included in a phylogeny, it would be premature to make further taxonomic adjustments based on this incomplete dataset. Monophyly at generic level cannot be ensured unless all taxa are included in the analysis.

Geographically, H. koelmaniorum occurs closest to the species in Haworthiopsis sect. Limifoliae (Smith 1950b: 3) Gildenhuys \& Klopper comb. nov. and sect. Tessellatae Salm-Dyck (1836: sect. 8) Gildenhuys \& Klopper comb. nov., but it is nonetheless well separated from both. Although populations of $H$. koelmaniorum occurs geographically closest to those in sect. Limifoliae, it has much more morphological similarities with sect. Tessellatae and appears to have a closer relationship with it. Thus far the phylogenetic affinities of these plants are insufficiently known and as H. koelmaniorum cannot be placed in any other section with certainty, its own section is established here. In due course, further evidence may suggest that the species belonging to the Haworthiopsis sect. Koelmaniorum Gildenhuys \& Klopper sect. nov. and sect. Tessellatae are better classified as a subgenus of Haworthiopsis or even in a genus of their own. The latter option might resolve the issue of non-monophyly in the current classification, but is not a viable option based on the available phylogeny of Manning et al. (2014). Inclusion of all species in these two sections in further molecular analyses might provide better resolution and determine the true affinities of these taxa. 


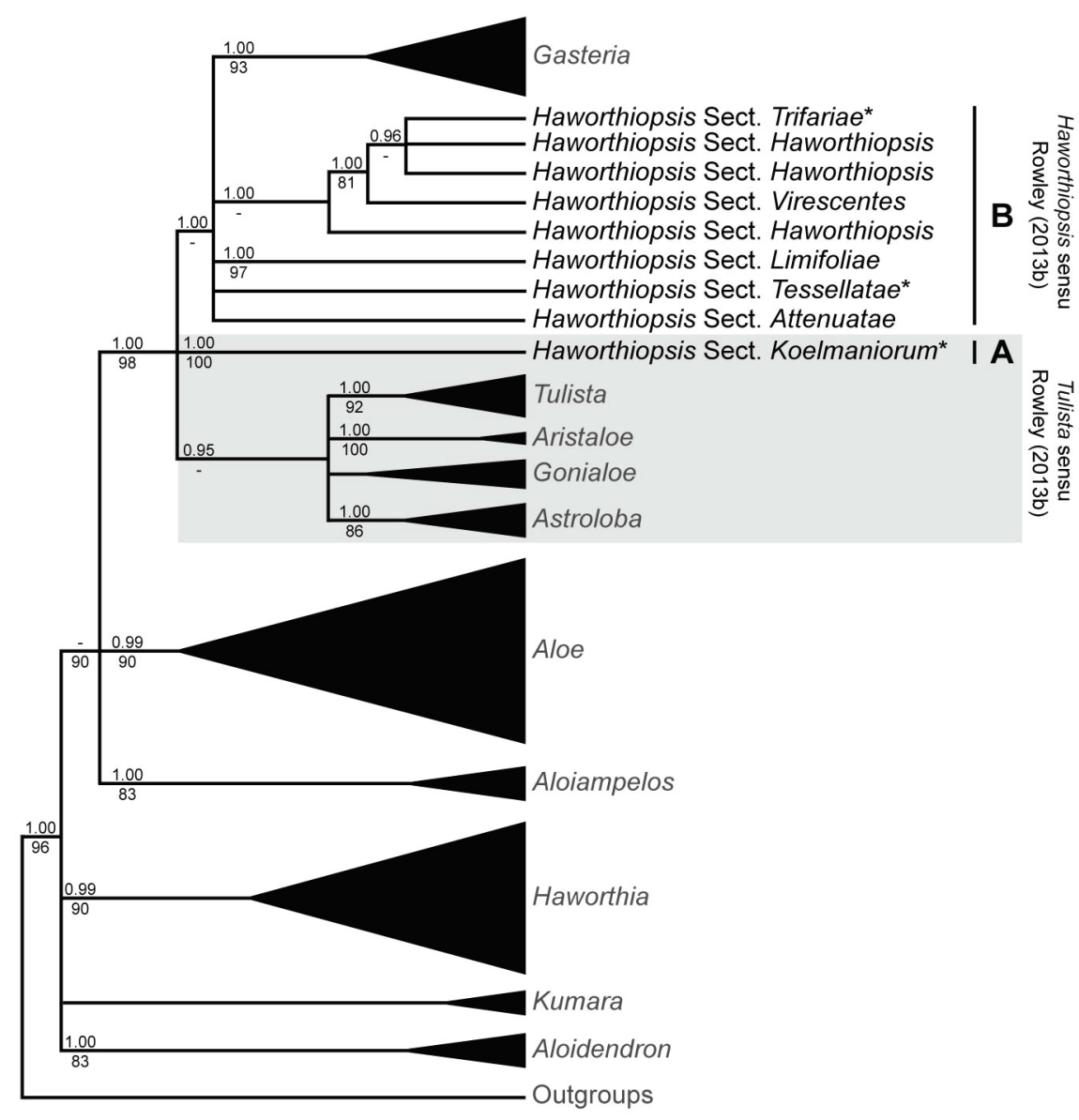

FIGURE 2. Phylogram of the Alooideae (adapted from Manning et al. 2014). Maximum parsimony bootstrap support is shown below the branches and Bayesian posterior probabilities above the branches. Only values indicating good support (bootstrap above 70\%, Bayesian posterior probabilities above 0.95 ) are shown. All unsupported branches were collapsed here. An asterisk $(*)$ indicates sections of Haworthiopsis where not all the members were included in the molecular analysis of Manning et al. (2014).

Haworthiopsis limifolia is recovered in a well-supported clade within the Haworthiopsis polytomy (Fig. 2). Geographically H. limifolia occurs well separated from the species of the type section, Haworthiopsis sect. Haworthiopsis, but morphologically it is similar to the species in this section. It is possible that this species belongs to the sect. Haworthiopsis, but for the time being, until its placement can be established through further studies, it is recognised in its own section, Haworthiopsis sect. Limifoliae as previously established by Smith (1950b). Haworthiopsis sect. Haworthiopsis as circumscribed here is the only section that is not monophyletic based on the phylogram of Manning et al. (2014). The inclusion of the remaining species of $H$. sect. Trifariae that were not investigated by Manning et al. (2014) will likely change the topology of the phylogram and hopefully provide better resolution within this clade. Once a robust phylogeny including all Haworthiopsis taxa is available, the sectional classification of Haworthiopsis, as proposed here, could be re-evaluated.

\section{Taxonomic changes on species and infraspecific level in Haworthiopsis}

Bayer (1999) was against the approach of Gerald Graham Smith (1892-1976) who prolifically described and published many new species, varieties and forms of Haworthia s.l. Indeed, Bayer (1982) also noted that Smith had intended to describe many more. Many of Smith's taxa and those from other relevant authors are not necessarily viable at species level as intended, but many of these are very significant and distinguishable forms or varieties that are worthy of recognition at infraspecific level. Polymorphism is encountered in Haworthiopsis, so that individuals in some populations deviate from the norm in one respect or another, but this sort of natural variation within a population should not give rise to separate taxonomic categories. However, where the majority of individuals within a population from a geographically separated area deviate from the norm of the species in some easily distinguishable way, there are certainly grounds for recognizing a subordinate taxon. In this treatment we follow established concepts for the 
recognition of infraspecific taxa at the level of varieties and formas as used by, for instance, Bayer \& Manning (2012).

As mentioned previously, Rowley (2013a) made certain nomenclatural mistakes when he published the new combinations in Haworthiopsis. Based on the basionym Aloe viscosa Linnaeus (1753: 460), Rowley (2013a) simultaneously proposed new combinations in both Haworthiopsis and Tulista in the new classification offered for the haworthioid taxa (published on 9 April 2013). According to Art. 36(2) of the International Code of Nomenclature for Algae, Fungi and Plants (ICN) (McNeill et al., 2012), neither of these combinations are validly published. Manning et al. (2014: 70) assumed that the entry under Tulista was a mistake by Rowley (2013a) and listed only the invalid combination made by Rowley (2013a) in Haworthiopsis. However, Rowley (2013b) had previously corrected his mistake and only included the combination in Tulista in the amended version of the classification (published on 2 August 2013), thus validating that combination. It is important to note here that no samples of "Tulista/Haworthiopsis viscosa" was included in the molecular analysis of Manning et al. (2014). Placement of this taxon in Tulista is contrary to the floral and foliar morphology of these plants and we concur with its placement in Haworthiopsis by Manning et al. (2014). Hence, the new combination for Aloe viscosa in Haworthiopsis is published here.

Similarly, Rowley (2013a) simultaneously proposed two new combinations for the basionym Haworthia granulata, firstly as Haworthiopsis granulata, followed by Haworthiopsis venosa var. granulata. Neither of these combinations in Rowley (2013a) are validly published [McNeill et al., 2012, Art. 36(2)]. In the amended version of this classification, Rowley (2013b) validated the combination Haworthiopsis granulata by omitting Haworthiopsis venosa var. granulata. He also added the new combination Haworthiopsis tessellata, the publication of which predates that of Manning et al. (2014) and their new combination for "Haworthiopsis tessellata (Haw.) Boatwr. \& J.C.Manning" in Manning et al. (2014: 71) is thus superfluous.

Rowley (2013a \& b) only recognised Haworthiopsis reinwardtii (Salm-Dyck 1821: 37) Rowley (2013a: 5) var. brevicula (Smith 1944a: 11) Rowley (2013a: 5), but not the formae previously established under var. reinwardtii by Bayer $(1976,1982$, 1999). Three of the formae previously accepted under Haworthia reinwardtii are here transferred and recognised as formae of Haworthiopsis reinwardtii and the new combinations are provided. The former Haworthia reinwardtii f. zebrina (Smith 1944a: 18) Bayer (1976: 169), which may be only worthy of recognition for horticultural purposes, applies to selected clones of f. olivacea (Smith 1944a: 16) Bayer (1976: 142) and is here included in its synonymy.

\section{Taxonomic setting}

Haworthiopsis Rowley (2013a: 4)

Type:-Haworthiopsis coarctata (Haw.) Rowley (2013a: 4) 三 Haworthia coarctata Haworth (1824: 301)= Haworthia sect. Hexangulares Uitewaal (1947a: 136) p.p. 三 Haworthia subg. Hexangulares (Uitewaal) Bayer (1971: 160)

Detailed description:-Plants dwarf, succulent perennial herbs, solitary to proliferating, acaulescent or caulescent to ca. $400 \mathrm{~mm}$ long. Leaves rosulate, arranged in ranks of three or five, or spirally inserted along the length of the stem, 10-350 mm long, and 6-40 mm wide near the base. Leaf surfaces viscid in some, glabrous to scabrous, tuberculate or with ridges, tubercles concolorous to white, in some the upper surfaces windowed, lined and often reticulated. Margins smooth, tuberculate, ridged or with cartilaginous teeth. Inflorescences with few sterile bracts, usually racemose, rarely paniculate. Perianth $<17 \mathrm{~mm}$ long, bilabiate, straight or curved, hexangular or rounded-hexangular at base, tapering to pedicel, white with brownish, pinkish or greenish hues or nerves, 3 upper tepals of the bilabiate perianth spreading to recurved, 3 lower tepals generally strongly recurved, inner and outer tepals joined at the base, rarely fused halfway, both whorls adhering. Anthers included. Style straight, included. Fruit a capsule $<24 \mathrm{~mm}$ long, narrowly ovoid. Seed $<4 \mathrm{~mm}$ long, usually black, to dark brown.

Identification key to the sections of Haworthiopsis (see Table 2 for a list of species included in the various sections)

1. Leaves rosulate, arranged in a caulescent to acaulescent spiral, or occasionally in a seemingly quinquefarious caulescent spiral; Eastern Cape, Western Cape, Northern Cape, Free State, North-West and Namibia 
- $\quad$ Leaves significantly arranged in a trifarious caulescent spiral or in three tiers, or in an acaulescent, quinquefarious twisted spiral; Eastern Cape and Western Cape .......................................................................................................... H. sect. Trifariae (F)

2. Leaves with opaque to translucent windowed areas on upper surface …......................................................................... 3

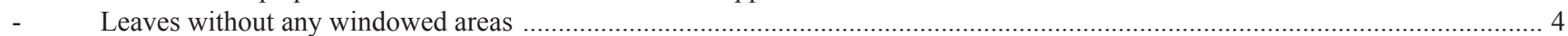

3. Leaves ovate-lanceolate to ovate-deltoid, upper surfaces with translucent windowed areas, leaf tips acuminate; Eastern Cape,

Western Cape, Northern Cape, Free State, North-West and Namibia ................................................................................ 5

Leaves navicular, upper surfaces with a truncated opaque to translucent end-area, leaf tips obtuse; Eastern Cape

H. sect. Virescentes $(\mathrm{G})$

4. Plants proliferating from the base or by stolons, acaulescent to caulescent; leaves incurved, erect to spreading, variable in colour;

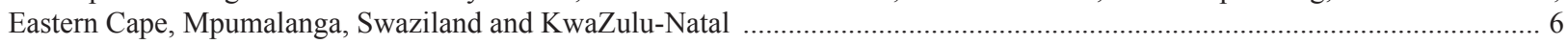
Plants solitary (rarely offsetting), acaulescent; leaves erect to spreading or recurved, dark green to blackish green; Eastern Cape .

H. sect. Virescentes $(\mathrm{G})$

5. Plants usually proliferous from base or by stolons, acaulescent to caulescent; upper leaf surfaces smooth; Eastern Cape, Western Cape, Northern Cape, Free State, North-West and Namibia ...................................................................... H. sect. Tessellatae (E) Plants mostly solitary, acaulescent; upper leaf surfaces pellucid; Mpumalanga .................................. H. sect. Koelmaniorum (C)

6. Plants proliferous from the base, acaulescent to caulescent; leaves incurved, erect to occasionally spreading; Eastern Cape ....... 7

- $\quad$ Plants stoloniferous, acaulescent; leaves spreading; Mpumalanga, Swaziland and KwaZulu-Natal ............. H. sect. Limifoliae (D)

7. Upper leaf surfaces usually without any tubercles, lower leaf surfaces glabrous to tuberculated, leaves firm and rigid, fibrous; plants acaulescent to caulescent; inflorescences usually simple (rarely branched) ............................... H. sect. Haworthiopsis (A) Upper leaf surfaces sparsely to densely tuberculated, lower leaf surfaces generally tuberculated, leaves softer and with viscid strands only; plants acaulescent; inflorescences usually branched H. sect. Attenuatae (B)

TABLE 2. The sections of Haworthiopsis recognised in this treatment and the species included in each section.

\begin{tabular}{|c|c|}
\hline Section & Species \\
\hline Attenuatae & H. attenuata \\
\hline \multirow[t]{5}{*}{ Haworthiopsis } & H. coarctata \\
\hline & H. fasciata \\
\hline & H. glauca \\
\hline & H. longiana \\
\hline & H. reinwardtii \\
\hline Limifoliae & H. limifolia \\
\hline Koelmaniorum & H. koelmaniorum \\
\hline \multirow[t]{4}{*}{ Tessellatae } & H. granulata \\
\hline & H. tessellata \\
\hline & H. venosa \\
\hline & H. woolleyi \\
\hline \multirow[t]{4}{*}{ Trifariae } & H. pungens \\
\hline & H. nigra \\
\hline & H. scabra \\
\hline & H. viscosa \\
\hline \multirow[t]{2}{*}{ Virescentes } & H. bruynsii \\
\hline & H. sordida \\
\hline
\end{tabular}

\section{A. Haworthiopsis sect. Haworthiopsis}

$\equiv$ Haworthia sect. Coarctatae Berger (1908: 75). Type:-Haworthiopsis coarctata (Haw.) Rowley (2013a: 4) $\equiv$ Haworthia coarctata Haworth (1824: 301)

\section{Haworthiopsis coarctata (Haw.) Rowley (2013a: 4)}

Bas.:- Haworthia coarctata Haworth (1824: 301) 三 Aloe coarctata (Haw.) Roemer \& Schultes (1829: 647) $\equiv$ Haworthia reinwardtii subsp. coarctata (Haw.) Halda (1997: 40). Type (neotype designated by Breuer \& Metzing 1997: 5):-SOUTH AFRICA. Eastern Cape: 16 km from Grahamstown to Bathurst [QDS: 3326DA], 1947, G.G. Smith 7092 (NBG0068473!) 
Distribution:- It is predominantly found in the area south of Grahamstown, from near the Fish River in the east to near Port Elizabeth in the west, Eastern Cape, South Africa. A few collections have also been made to the north of Grahamstown.

\section{1a. var. coarctata}

= Haworthia greenii Baker (1880: 202) 三 Haworthia coarctata var. greenii (Baker) Bayer (1973: 80) E Haworthia reinwardtii var. greenii (Baker) Halda (1997: 41) E Haworthia coarctata f. greenii (Baker) Bayer (1999: 172).

= Haworthia peacockii Baker (1880: 202)

= Haworthia chalwinii Marloth \& Berger (1906: 247) E Haworthia reinwardtii var. chalwinii (Marloth \& A.Berger) Resende (1943: 80) $\equiv$ Haworthia coarctata f. chalwinii (Marloth \& A.Berger) Pilbeam (1983: 52)

= Haworthia fallax Von Poellnitz (1933: 83) 三 Haworthia reinwardtii var. fallax (Poelln.) Von Poellnitz (1937: 209a)

= Haworthia reinwardtii var. conspicua Von Poellnitz (1937: 210a) E Haworthia coarctata f. conspicua (Poelln.) Pilbeam (1983: 52)

= Haworthia reinwardtii var. pseudocoarctata Von Poellnitz (1940a: 43) $\equiv$ Haworthia coarctata f. pseudocoarctata (Poelln.) Resende (1943: 84)

= Haworthia coarctata var. haworthii Resende (1943: 84)

= Haworthia coarctata var. kraussii Resende (1943: 84)

= Haworthia greenii f. bakeri Resende (1943: 87)

= Haworthia greenii f. minor Resende (1943: 87)

= Haworthia reinwardtii var. committeesensis Smith (1943: 93)

= Haworthia fulva Smith (1943: 103)

= Haworthia greenii var. silvicola Smith (1943: 103)

= Haworthia baccata Smith (1944a: 20)

= Haworthia reinwardtii var. huntsdriftensis Smith (1944a: 14)

= Haworthia musculina Smith (1948: 49)

[“Haworthia coarctatoidea Resende \& Lopez” (1948: 176) nom. nud.]

Distribution:- The typical variety is found over most of the distribution range of the species.

1b. var. adelaidensis (Poelln.) Rowley (2013a: 4)

Bas.:-Haworthia reinwardtii var. adelaidensis Von Poellnitz (1940a: 43) 三 Haworthia coarctata subsp. adelaidensis (Poelln.) Bayer (1973: 86) $\equiv$ Haworthia coarctata var. adelaidensis (Poelln.) Bayer (1999: 172) E Haworthia adelaidensis (Poelln.) Breuer (2010: 7). Type (lectotype designated by Breuer \& Metzing 1997: 5):- (unpublished icon) "Haworthia reinwardtii Haw. var. adelaidensis v.P." (B).

= Haworthia reinwardtii var. riebeeckensis Smith (1944a: 16)

= Haworthia reinwardtii var. bellula Smith (1945: 70) $\equiv$ Haworthia coarctata f. bellula (G.G.Sm.) Pilbeam (1983: 52)

Distribution:- It is found in the area north and west of Grahamstown, Eastern Cape, South Africa.

1c. var. tenuis (G.G.Sm.) Rowley (2013a: 4)

Bas.:-Haworthia reinwardtii var. tenuis Smith (1948: 51) 三 Haworthia coarctata subsp. coarctata var. tenuis (G.G.Sm.) Bayer (1973: 80) $\equiv$ Haworthia tenuis (G.G.Sm.) Breuer (2010: 8). Type:-SOUTH AFRICA. Eastern Cape: Farm Harvestvale, near Alexandria [QDS: 3326CB], 1940, G.G. Smith 3420 (holotype NBG0112024!; isotype NBG).

Distribution:- It is only known from near Alexandria, Eastern Cape, South Africa.

2. Haworthiopsis fasciata (Willd.) Rowley (2013a: 4)

Bas.:-Apicra fasciata Willdenow (1811: 270) 三 Haworthia fasciata (Willd.) Haworth (1819: 57) 三 Aloe fasciata (Willd.) Salm-Dyck (1834: 326) $\equiv$ Haworthia pumila subsp. fasciata (Willd.) Halda (1997: 37). Type (neotype designated by Breuer \& Metzing 1997: 6):-SOUTH AFRICA. Eastern Cape: Hankey [QDS: 3324DA], 1962, F.J. Stayner s.n. (NBG0110360!). 
Distribution:- It is known from Kareedouw in the west, to Port Elizabeth in the east and northwards to north of Uitenhage, Eastern Cape, South Africa.

\section{2a. var. fasciata}

= Aloe fasciata var. major Salm-Dyck (1837: t.158) E Haworthia fasciata var. major (Salm-Dyck) Haworth (1821: 54)

= Haworthia fasciata var. subconfluens Von Poellnitz (1937: 133b) 三 Haworthia fasciata f. subconfluens (Poelln.) Von Poellnitz (1938a: 95)

= Haworthia fasciata f. ovato-lanceolata Von Poellnitz (1938a: 96)

= Haworthia fasciata f. sparsa Von Poellnitz (1938a: 96)

= Haworthia fasciata f. variabilis Von Poellnitz (1938a: 96)

= Haworthia fasciata f. vanstaadenensis Von Poellnitz (1938a: 97)

Distribution:- The typical variety is found across most of the range of distribution for the species, it occurs from near Kareedouw in the west to near Port Elizabeth in the east, northward at Uitenhage, Eastern Cape, South Africa.

2b. var. browniana (Poelln.) Gildenh. \& Klopper comb. nov.

Bas.:-Haworthia browniana Von Poellnitz (1937c: 102) 三 Haworthia fasciata f. browniana (Poelln.) Bayer (1976: 105) 三 Haworthia fasciata var. browniana (Poelln.) Scott (1985: 21). Type (lectotype designated by Breuer \& Metzing 1997: 7):- (photographic icon) "Haworthia browniana v.P”. (B); later published in Von Poellnitz (1937c: 102).

Distribution:- It is only known from the type locality northwest of Uitenhage, Eastern Cape, South Africa.

\section{Haworthiopsis glauca (Baker) Rowley (2013a: 4)}

Bas.:- Haworthia glauca Baker (1880: 203) 三 Haworthia reinwardtii subsp. glauca (Baker) Halda (1997: 41). Type:- SOUTH AFRICA. Cape: Without locality, 1862, T. Cooper s.n. (holotype K0256759!). Cited by Scott (1985).

Distribution:- It generally occurs further inland and towards the west than the other stem-forming species in this section. It is reported from near Uitenhage in the east to near Willowmore in the west, Eastern Cape, South Africa.

\section{3a. var. glauca}

= Haworthia carrissoi Resende (1941: 161)

Distribution:- The typical variety is reported from the Zuurberg Pass in the east to near Willowmore in the west, Eastern Cape, South Africa.

3b. var. herrei (Poelln.) Rowley (2013a: 4)

Bas.:- Haworthia herrei Von Poellnitz (1929a: 24) 三 Haworthia glauca var. herrei (Poelln.) Bayer (1976: 122) 三 Haworthia reinwardtii var. herrei (Poelln.) Halda (1997: 41). Type (neotype designated by Breuer \& Metzing 1997: 7):-SOUTH AFRICA. Eastern Cape: Campherspoort [QDS: 3324AA], 1947, W.F. Barker 5069 (NBG0068132!).

= Haworthia herrei Poelln. var. depauperata Von Poellnitz (1933: 86)

= Haworthia herrei Poelln. var. poellnitzii Resende (1941: 164) nom. illeg.

= Haworthia armstrongii Von Poellnitz (1937d: 152) 三 Haworthia glauca f. armstrongii (Poelln.) Bayer (1976: 99)

= Haworthia jacobseniana Von Poellnitz (1937c: 102) 三 Haworthia glauca f. jacobseniana (Poelln.) Pilbeam (1983: 74)

= Haworthia eilyae Von Poellnitz (1937d: 152)

= Haworthia eilyae Poelln. var. poellnitziana Resende (1943: 89) nom. illeg.

= Haworthia eilyae Poelln. var. zantneriana Resende (1943: 90)

= Haworthia jonesiae Von Poellnitz (1937d: 153) E Haworthia glauca f. jonesiae (Poelln.) Pilbeam (1983: 74) 
Distribution:- This variety is known from northwest of Uitenhage, westwards to the area northeast of Willowmore, Eastern Cape, South Africa.

\section{Haworthiopsis longiana (Poelln.) Rowley (2013a: 4)}

Bas.:-Haworthia longiana Von Poellnitz (1937a: 203) 三 Haworthia pumila subsp. longiana (Poelln.) Halda (1997: 37). Type (lectotype designated by Breuer \& Metzing 1997: 8):- (photographic icon), “Haworthia longiana v.P”(B).

= Haworthia longiana Poelln. var. albinota Smith (1948: 44)

Distribution:- It has a limited distribution in the Gamtoos River Valley, predominantly from north of Humansdorp, around Hankey and Patensie, Eastern Cape, South Africa.

\section{Haworthiopsis reinwardtii (Salm-Dyck) Rowley (2013a: 5)}

Bas.:-Aloe reinwardtii Salm-Dyck (1821: 37) 三 Haworthia reinwardtii (Salm-Dyck) Haworth (1821: 53). Type (lectotype designated by Scott 1981a: 36):- (icon) in Salm-Dyck (1836: Aloe t.12 [sect. 6: 16]). Type (epitype designated by Breuer \& Metzing 1997: 10):-SOUTH AFRICA. Eastern Cape: Near top of hill above Ncera River Bridge [QDS: 3327BA], 1940, G.G. Smith 3563 (NBG0068556).

Distribution:- It is found over a relatively narrow range in the Eastern Cape, South Africa, from near East London in the east to near the Bushman's River in the west.

\section{5a. var. reinwardtii f. reinwardtii}

= Haworthia reinwardtii var. major Baker (1880: 202)

= Haworthia reinwardtii var. pulchra Von Poellnitz (1937: 209a)

= Haworthia reinwardtii var. archibaldiae Von Poellnitz (1937: 210a)

= Haworthia reinwardtii var. peddiensis Smith (1943: 94)

= Haworthia reinwardtii var. haworthii Resende (1943: 79)

= Haworthia reinwardtii var. triebneri Resende (1943: 80)

= Haworthia reinwardtii var. valida Smith (1943: 98)

= Haworthia reinwardtii var. grandicula Smith (1944a: 12)

Distribution:- The typical variety is found across the range of distribution for the species, mainly along the lower Keiskamma and Great Fish River catchment areas. However, it is also recorded from west of East London in the east to near the Bushman's River in the west, and inland from near Peddie towards Grahamstown, Eastern Cape, South Africa.

5b. var. reinwardtii f. chalumnensis (G.G.Sm.) Gildenh. \& Klopper comb. nov.

Bas.:-Haworthia reinwardtii var. chalumnensis Smith (1943: 99) 三 Haworthia reinwardtii f. chalumnensis (G.G.Sm.) Bayer (1976: 106). Type:-SOUTH AFRICA. Eastern Cape: on the banks of the Chalumna River, 48.3 km west of East London [QDS: 3327BA], 1934, G.G. Smith 513 (holotype NBG0068486!; isotype NBG, PRE).

Distribution:-Although various plants occurring near Chalumna and the adjacent Tyolumnqua River (Eastern Cape, South Africa) are often considered as this form, it appears as if the typical robust form is only found from near the Chalumna police station.

5c. var. reinwardtii f. kaffirdriftensis (G.G.Sm.) Gildenh. \& Klopper comb. nov.

Bas.:-Haworthia reinwardtii var. kaffirdriftensis Smith (1943: 12) 三 Haworthia reinwardtii f. kaffirdriftensis (G.G.Sm.) Bayer (1976: 126). Type:-SOUTH AFRICA. Eastern Cape: near Kaffirdrift, eastern side of Fish River, about $22 \mathrm{~km}$ south south west of Peddie [QDS: 3327AC], 1940, G.G. Smith 3364 (holotype NBG0068355!; isotype NBG0112001!, PRE0592668!). 
Distribution:- It is restricted to a small population, found east of Kaffirdrift on the eastern bank of the Great Fish River, Eastern Cape, South Africa.

5d. var. reinwardtii f. olivacea (G.G.Sm.) Gildenh. \& Klopper comb. nov.

Bas.:- Haworthia reinwardtii var. olivacea Smith (1944a: 16) $\equiv$ Haworthia reinwardtii f. olivacea (G.G.Sm.) Bayer (1976: 142) $\equiv$ Haworthia olivacea (G.G.Sm.) Breuer (2010: 7). Type:-SOUTH AFRICA. Eastern Cape: South east of Kaffirdrift, Peddie District [QDS: 3327AC], 1942, G.G. Smith 5260 (holotype NBG0112000!; isotype NBG, PRE0592641!).

= Haworthia reinwardtii var. zebrina Smith (1944a: 18) E Haworthia reinwardtii f. zebrina (G.G.Sm.) Bayer (1976: 169)

Distribution:- It occurs on both the western and eastern banks, in the vicinity of Kaffirdrift next to the Great Fish River, Eastern Cape, South Africa.

5e. var. brevicula (G.G.Sm.) Rowley (2013a: 5)

Bas.:-Haworthia reinwardtii var. brevicula Smith (1944a: 11) 三 Haworthia brevicula (G.G.Sm.) Breuer (2010: 7). Type:-SOUTH AFRICA. Eastern Cape: Between Frazer's Camp and the old fort, Albany Division [QDS: 3326BD], 1940, G.G. Smith 3138 (holotype NBG0112112!; isotype NBG0068168!, PRE0834962!).

= Haworthia reinwardtii var. diminuta Smith (1948: 52)

Distribution:- It has a rather limited distribution and is known from a few scattered populations along the Kapp River Valley to the Great Fish River, Eastern Cape, South Africa.

B. Haworthiopsis sect. Attenuatae (Pilbeam) Gildenh. \& Klopper comb. nov.

Bas.:-Haworthia subsect. Attenuatae Pilbeam (1983: 15) 三 Haworthia ser. Attenuatae (Pilbeam) Rowley (1985: 14) $\equiv$ Haworthia sect. Attenuatae (Pilbeam) Breuer (2010: 4). Type (lectotype designated here):-Haworthiopsis attenuata (Haw.) Rowley (2013a: 4) $\equiv$ Aloe attenuata Haworth (1804: 11)

6. Haworthiopsis attenuata (Haw.) Rowley (2015: 2)

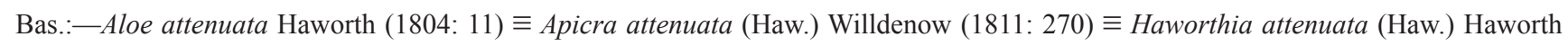
(1812: 92) $\equiv$ Haworthia pumila subsp. attenuata (Haw.) Halda (1997: 36) [“Haworthiopsis attenuata (Haw.) Rowley” (2013a: 4) nom. inval., Art. 41.8.a (McNeill et al., 2012)]. Type (neotype designated by Breuer \& Metzing 1997: 4): SOUTH AFRICA. Eastern Cape: Sandland, $20 \mathrm{~km}$ east of Patensie [QDS: 3324DA], P.L. Perry 660 (NBG0144672!).

Distribution:- Plants vary over their wide range of distribution in the Eastern Cape, South Africa, from the Gamtoos River Valley in the west to the Bashee (Mbashe) River Valley in the east.

6a. var. attenuata

= Haworthia radula var. magniperlata Haworth (1821: 54)

= Haworthia clariperla Haworth (1828: 186) 三 Aloe attenuata var. clariperla (Haw) Salm-Dyck (1834: 12b) E Haworthia attenuata var. clariperla (Haw.) Baker (1880: 204) 三 Haworthia attenuata f. clariperla (Haw.) Pilbeam (1983: 45)

= Aloe subulata Salm-Dyck (1829: 1712) 三 Haworthia subulata (Salm-Dyck) Baker (1880: 206)

= Aloe rugosa Salm-Dyck (1834: 323) 三 Haworthia rugosa (Salm-Dyck) Baker (1880: 206)

= Aloe rugosa var. laetevirens Salm-Dyck (1834: 323)

= Aloe rugosa var. perviridis Salm-Dyck (1834: 323) E Haworthia rugosa var. perviridis (Salm-Dyck) Berger (1908: 92)

= Haworthia britteniana Von Poellnitz (1937: 196a) $\equiv$ Haworthia attenuata var. britteniana (Poelln.) Von Poellnitz (1937: 166e) $\equiv$ Haworthia attenuata f. britteniana (Poelln.) Bayer (1982: 63)

= Haworthia attenuata var. o'donoghueana Farden (1939: 34)

= Haworthia attenuata var. deltoidea Farden (1939: 36)

= Haworthia attenuata var. linearis Farden (1939: 36)

= Haworthia attenuata var. minissima Farden (1939: 37) 
[“Haworthia attenuata var. uitewaaliana Farden” (1939: 36) nom. inval. (Art. 39.1, McNeill et al., 2012)]

[“Haworthia attenuata var. inusitata Farden" (1939: 38) nom. inval. (Art. 39.1, McNeill et al., 2012)]

Distribution:-The typical variety is the most widespread, and is found in the central and western part of the distribution range of the species.

6b. var. glabrata (Salm-Dyck) Rowley (2015: 2)

Bas.:- Aloe glabrata Salm-Dyck (1834: 325) 三 Haworthia glabrata (Salm-Dyck) Baker (1880: 206) 三 Haworthia attenuata var. glabrata (Salm-Dyck) M.B.Bayer in Bayer \& Manning (2012: 37) [“Haworthiopsis attenuata var. glabrata (Salm-Dyck) Rowley" (2013a: 4) nom. inval. (Art. 35.1, McNeill et al., 2012)]. Type (neotype designated by Smith \& Greyling 1990: 332):-(icon) in Salm-Dyck (1840: Aloe t.7).

= Aloe glabrata var. concolor Salm-Dyck (1849: Aloe t.2) E Haworthia glabrata var. concolor (Salm-Dyck) Baker (1880: 206)

= Aloe glabrata var. perviridis Salm-Dyck (1849: Aloe t.1) E Haworthia glabrata var. perviridis (Salm-Dyck) Baker (1880: 206)

Distribution:- Although it has been known in cultivation for a very long time, it was never known from any wild populations until Bayer (1999) linked this taxon to the interesting material collected by Peter Bruyns from the Bashee River, Eastern Cape, South Africa.

6c. var. radula (Jacq.) Rowley (2015: 2)

Bas.:-Aloe radula Jacquin (1804: 11) 三 Apicra radula (Jacq.) Willdenow (1811: 270) 三 Haworthia radula (Jacq.) Haworth (1812: 93) 三 Haworthia attenuata var. radula (Jacq.) Bayer (1999: 167) 三 Haworthia pumila subsp. radula (Jacq.) Halda (1997: 37) [“Haworthiopsis attenuata var. radula (Jacq.) Rowley" (2013a: 4) nom. inval. (Art. 35.1, McNeill et al., 2012)]. Type (lectotype designated by Scott 1985: 18):- (icon) in Jacquin (1804: t.422). Type (epitype designated by Breuer \& Metzing 1997: 10):-SOUTH AFRICA. Eastern Cape: 1.6 km from Hankey to Thornhill [QDS: 3324DD], 1940, G.G. Smith 3190 (NBG0115013!).

= Aloe radula var. major Salm-Dyck (1817: 4)

= Aloe radula var. media Salm-Dyck (1817: 4)

= Aloe radula var. minor Salm-Dyck (1817: 4)

= Haworthia radula var. asperior Haworth (1821: 54)

= Haworthia radula var. laevior Haworth (1821: 54)

= Haworthia radula var. pluriperlata Haworth (1821: 54)

Distribution:- This variety is limited in distribution and found in the area of Hankey, Eastern Cape, South Africa.

\section{Haworthiopsis sect. Koelmaniorum Gildenh. \& Klopper sect. nov.}

Haworthiopsis G.D.Rowley sect. Koelmaniorum Gildenh. \& Klopper Sectio nova Tessellatis affinis sed habitu solitario, depresso, non sobolifero, radicibus valde incrassatis, foliis rigidis, acutis usque triangulatis, supra planis vel convexis, obscure pellucidis, tuberculatis, tuberculis obscure translucentibus, subtus tuberculis irregularibus concoloribus, saepe in cristas transversas confluentibus, differt.

Type:- Haworthiopsis koelmaniorum (Oberm. \& Hardy) Boatwr. \& J.C.Manning in Manning et al. (2014: 70) 三Haworthia koelmaniorum Obermeyer \& Hardy (1967: t. 1502)

Affinities with sect. Tessellatae. Plants solitary, rarely dividing, rosulate, acaulescent. Roots swollen. Leaves spirally disposed, spreading, firm, acuminate to triangular; upper surfaces flattened to convex, windowed, translucence opaque, with raised translucent bumps, lined inside the window, occasionally connecting transversely; lower surfaces with raised concolorous tubercles often forming transverse ridges; margins with raised cartilaginous tubercles occasionally becoming teeth. Inflorescence simple gracile raceme. Flowers slender and smaller than usually found, tepals fused about halfway towards the base, base rounded-hexangular.

7. Haworthiopsis koelmaniorum (Oberm. \& D.S.Hardy) Boatwr. \& J.C.Manning in Manning et al. (2014: 14)

Bas.:-Haworthia koelmaniorum Obermeyer \& Hardy (1967: t.1502) 三 Haworthia limifolia subsp. koelmaniorum (Oberm. \& D.S.Hardy) Halda (1997: 38) $\equiv$ Tulista koelmaniorum (Oberm. \& D.S.Hardy) Rowley (2013a: 5). Type:-SOUTH AFRICA. Mpumalanga: 
Near Groblersdal, on north facing slopes in sandstone ridges [QDS: 2529AB], 1966, D.S. Hardy \& A.A. Mauve 2267 (holotype PRE0037965!).

Distribution:- This is among the northernmost occurring Haworthiopsis species, and is known from a small area in both Limpopo and Mpumalanga, South Africa.

\section{7a. var. koelmaniorum}

Distribution:- It is found to the south of Groblersdal, to near Dennilton in the west and to near Maleoskop in the east, Limpopo, South Africa.

7b. var. mcmurtryi (C.L.Scott) Gildenh. \& Klopper stat. nov.

Bas.:- Haworthia mcmurtryi Scott (1984: 69) $\equiv$ Haworthia koelmaniorum var. mcmurtryi (C.L.Scott) Bayer (1999: 181) $\equiv$ Tulista koelmaniorum var. mcmurtryi (C.L.Scott) Rowley (2013a: 6) 三 Haworthiopsis mcmurtryi (C.L.Scott) Zonneveld (2014: 949). Type:-SOUTH AFRICA. Mpumalanga: South west of Loskop Dam [QDS: 2529AB], 1984, D. McMurtry 5247 in C.L. Scott 7682 (holotype PRE0747960!).

Distribution:- It occurs to the southwest of the typical variety, along a narrow stretch to the west and southwest of Loskop Dam, Mpumalanga, South Africa.

\section{Haworthiopsis sect. Limifoliae (G.G.Sm.) Gildenh. \& Klopper comb. nov.}

Bas:- Haworthia sect. Limifoliae Smith (1950b: 3). Type (designated by Scott 1985):- Haworthiopsis limifolia (Marl.) Rowley (2013a: 4) [=Haworthia limifolia Marloth (1910: 409)].

8. Haworthiopsis limifolia (Marl.) Rowley (2013a: 4)

Bas.:- Haworthia limifolia Marloth (1910: 409). Type:-SWAZILAND: West of Delagoa Bay [QDS: 2632AB], 1908, R. Marloth 4678 (holotype PRE0037944!).

Distribution:- This is one of only two Haworthiopsis species that are known to occur as far north as Mpumalanga, South Africa (the other being H. koelmaniorum that also extends into Limpopo). It is also the only species found in KwaZulu-Natal (South Africa), Swaziland and possibly also Mozambique.

\section{8a. var. limifolia}

= Haworthia limifolia var. diploidea Resende (1940: 200)

= Haworthia limifolia var. tetraploidea Resende (1940: 114)

= Haworthia limifolia f. marlothiana Resende (1941: 200) E Haworthia limifolia var. marlothiana (Resende) Resende (1943: 93)

= Haworthia limifolia var. schuldtiana Resende (1943: 93)

= Haworthia limifolia var. stolonifera Resende (1943: 94)

= Haworthia limifolia $\mathrm{f}$. pimentelli Resende (1943: 94)

= Haworthia limifolia f. major Resende (1943: 94) $\equiv$ Haworthia limifolia f. major (Resende) Pilbeam (1983: 87)

[“Haworthia limifolia var. striata Pilbeam” (1983: 87) nom. inval. (Art. 40.1, McNeill et al., 2012)]

Distribution:- It occurs from the Umfolozi River in KwaZulu-Natal (South Africa) in the south, northward through Swaziland and up to Barberton (Mpumalanga, South Africa) in the north.

8b. var. arcana (Gideon F.Sm. \& N.R.Crouch) Rowley (2013a: 4)

Bas.:- Haworthia limifolia var. arcana Smith \& Crouch (2001: 119) 三 Haworthia arcana (Gideon F.Sm. \& N.R.Crouch) Breuer (2010: 7). Type:-SOUTH AFRICA. Mpumalanga: Near Hectorspruit [QDS: 2531BC], N.R. Crouch \& G.F. Smith 7 (holotype PRE0500088!) 
Distribution:- It is currently only known with certainty from the type locality near Hectorspruit, Mpumalanga, South Africa.

8c. var. gigantea (M.B.Bayer) Rowley (2013a: 4)

Bas.:-Haworthia limifolia var. gigantea Bayer (1962: 215) 三 Haworthia gigantea (M.B.Bayer) Hayashi (2000: 13). Type:--SOUTH AFRICA. KwaZulu-Natal: Nongoma district [QDS: 2731DC], 1962, F. Bayer in M.B. Bayer 112 (holotype PRE0037946!).

Distribution:-Although it has been reported to have come from Nongoma (KwaZulu-Natal, South Africa), this can not be confirmed (Bayer 1999).

8d. var. glaucophylla (M.B.Bayer) Rowley (2013a: 5)

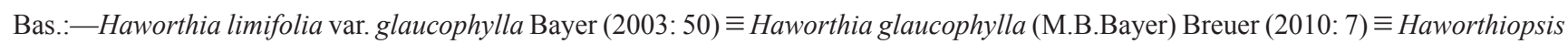
limifolia var. glaucophylla (M.B.Bayer) Rowley (2013a: 5). Type:-SOUTH AFRICA. Mpumalanga: Three Sisters [QDS: 2531CB], F. Venter 13700 (holotype NBG).

Distribution:- It is currently only known from Three Sisters, Mpumalanga, South Africa.

8e. var. ubomboensis (Verd.) Rowley (2013a: 5)

Bas.:-Haworthia ubomboensis Verdoorn (1941: t.818) 三 Haworthia limifolia var. ubomboensis (I.Verd.) Smith (1950b: 3). Type:SWAZILAND: 16 km. south of Stegi, Ubombo Mountains [QDS: 2632CA], 1937, D.R. Keith s.n. (holotype PRE0037954!).

= Haworthia limifolia var. keithii Smith (1950b: 4)

Distribution:- It is only known from the type locality, to the south of Stegi (Isiteki), eastern Swaziland.

E. Haworthiopsis sect. Tessellatae (Salm-Dyck) Gildenh. \& Klopper comb. nov.

Bas.:-Aloe sect. Tessellatae Salm-Dyck (1836: sect. 8) 三 Haworthia sect. Tessellatae (Salm-Dyck) Baker (1880: 199). Type (designated by Manning et al. 2014):- -Haworthiopsis tessellata (Haw.) Rowley (2013b: 5) 三 Haworthia tessellata Haworth (1824: 300).

= Haworthia sect. Venosae Berger (1908: 75)

9. Haworthiopsis granulata (Marloth) Rowley (2013b: 4)

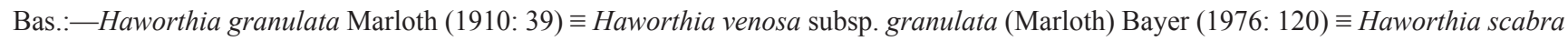
subsp. granulata (Marloth) Halda (1997: 35) [“Haworthiopsis granulata (Marloth) Rowley” (2013a: 4) nom. inval. (Art. 36.2, McNeill et al., 2012); Haworthiopsis venosa var. granulata (Marloth) Rowley” (2013a: 5) nom. inval. (Art. 36.2, McNeill et al., 2012)]. Type (neotype designated by Breuer \& Metzing 1997: 13):- SOUTH AFRICA. Northern Cape: Verlatenkloof, south of Sutherland [QDS: 3220AD], 1968, H. Hall 3168 (NBG0087961!).

= Haworthia schoemanii Hayashi (2003: 14)

Distribution:- It is known in the south from near Touwsrivier and Laingsburg (Western Cape) and northwards to near Sutherland (Northern Cape), South Africa.

10. Haworthiopsis tessellata (Haw.) Rowley (2013b: 5)

Bas.:- Haworthia tessellata Haworth (1824: 300) $\equiv$ Aloe tessellata (Haw.) Roemer \& Schultes (1829: 635) $\equiv$ Haworthia venosa subsp. tessellata (Haw.) Bayer (1976: 161) 三 Haworthia venosa var. tessellata (Haw.) Halda (1997: 39) [“Haworthiopsis tessellata (Haw.) Boatwr. \& J.C.Manning” in Manning et al. (2014: 71) nom. superfl.] Type (lectotype designated by Scott 1978: 75):-(icon) in (K) later published in Scott (1978: 75).

Distribution:- This species is the most widespread of all the species of Haworthiopsis. It is found in the drier interior, mostly summer rainfall areas, of five of the South African provinces (Eastern, Western and Northern Cape, Free State and North-West), as well as southern Namibia. 


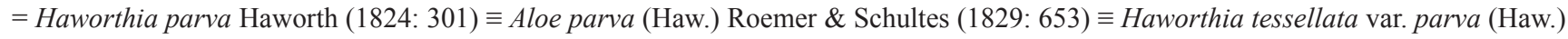
Baker (1880: 211)

= Haworthia tessellata var. inflexa Baker (1880: 211)

= Haworthia engleri Dinter (1914: 31) = Haworthia tessellata var. engleri (Dinter) Von Poellnitz (1938b: 202)

= Haworthia pseudotessellata Von Poellnitz (1929b: 133)

= Haworthia tessellata var. tuberculata Von Poellnitz (1937a: 214)

= Haworthia minutissima Von Poellnitz (1939a: 193) E Haworthia tessellata var. minutissima (Poelln.) Viveiros (1949: 200)

= Haworthia tessellata var. elongata Van Woerden (1940: 37)

= Haworthia tessellata var. simplex Resende \& Von Poellnitz (1942: 49)

= Haworthia tessellata var. stepheneana Resende \& Von Poellnitz (1942: 50)

= Haworthia tessellata var. luisierii Resende \& Von Poellnitz (1942: 51)

= Haworthia tessellata var. palhinhiae Resende \& Von Poellnitz (1942: 51)

= Haworthia tessellata var. velutina Resende \& Von Poellnitz (1942: 52)

= Haworthia tessellata var. obesa Resende \& Von Poellnitz (1942: 54)

= Haworthia tessellata var. coriacea Resende \& Von Poellnitz (1942: 53) EHaworthia coriacea (Resende \& Poelln.) Breuer (2010: 7)

= Haworthia tessellata f. longior Resende \& Von Poellnitz (1942: 53)

= Haworthia tessellata f. brevior Resende \& Von Poellnitz (1942: 53)

Distribution:- This widespread variety is known from the drier interior, mostly summer rainfall areas, of South Africa (Eastern, Western and Northern Cape, Free State and North-West), and southern Namibia.

10b. var. craussii (M.Hayashi) Gildenh. \& Klopper comb. et stat. nov.

Bas.:-Haworthia crausii Hayashi (2001: 16). Type:-SOUTH AFRICA. Northern Cape: Postmasburg [QDS 2823AC], M. Hayashi 97001 (holotype TUAT-RIEB).

Distribution:- This variety is known from the north eastern range of the distribution for this species. It is known from the Griekwastad, Postmasburg, Danielskuil and Riverton areas (Northern Cape).

\section{Haworthiopsis venosa (Lam.) Rowley (2013a: 4)}

Bas.:-Aloe venosa Lamarck (1783: 89) 三 Haworthia venosa (Lam.) Haworth (1812: 51). Type (lectotype designated by Scott 1987: 35):- (icon) in Commelijn (1703: t.29). Type (epitype designated by Breuer \& Metzing 1997: 13): SOUTH AFRICA. Western Cape: Swellendam, west of Breede River Bridge [QDS: 3420AB], 1970, M. B. Bayer 168 (NBG0110636).

= Aloe anomala Haworth (1804: 25) EApicra anomala (Haw.) Willdenow (1811: 273)

= Haworthia venosa var. oerthendahlii Hjelmquist (1943: 233)

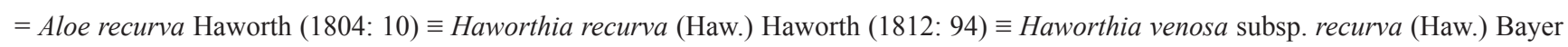
(1976: 149)

= Aloe tricolor Haworth (1804: 25)

= Haworthia distincta Brown (1876: 130)

Distribution:- It is predominantly known from rocky areas of the Breede River Valley to the south of Swellendam, Western Cape, South Africa. An odd population of plants has also been recorded from the Gouritz River Valley towards the east.

\section{Haworthiopsis woolleyi (Poelln.) Rowley (2013a: 5)}

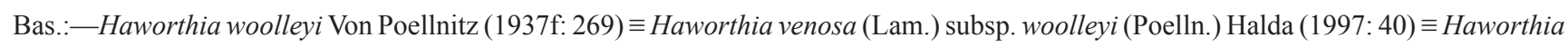
venosa (Lam.) subsp. woolleyi (Poelln.) Bayer (1999: 204) nom. superfl. Type (lectotype designated by Breuer \& Metzing 1997: 13):- (photographic icon) "Haworthia woolleyi v.P." (B), later published in Von Poellnitz (1938c: 3)

Distribution:- - It is restricted to a single and small population on a hill near Kleinpoort to the east of Steytlerville, an arid area of the Eastern Cape, South Africa. 


\section{F. Haworthiopsis sect. Trifariae (Haw.) Gildenh. \& Klopper comb. nov.}

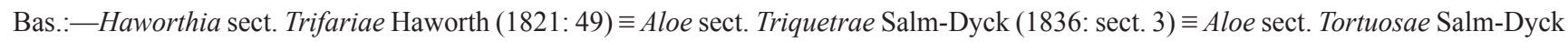
(1836: sect. 4). Type (designated by Scott 1985: 26):- Haworthiopsis viscosa (L.) Gildenh. \& Klopper, comb. nov. [=Aloe viscosa Linnaeus (1753: 322)].

= Haworthia sect. Luridae Haworth (1821: 50)

= Haworthia sect. Rigidae Haworth (1821: 49)

= Haworthia sect. Scabrae Berger (1908: 75) 三 Haworthia ser. Scabrae (A.Berger) Rowley (1985: 5)

13. Haworthiopsis pungens (M.B.Bayer) Boatwr. \& J.C.Manning in Manning et al. (2014: 14)

Bas.:-Haworthia pungens Bayer (1999: 188) 三 Tulista pungens (M.B. Bayer) Rowley (2013a: 6). Type:-SOUTH AFRICA. Eastern Cape: Braamrivier, Joubertina [QDS: 3323DD], P.V. Bruyns 7090 (holotype B).

Distribution:- It has a limited distribution and is currently only known from populations north of Joubertina and Kareedouw, Eastern Cape, South Africa.

\section{Haworthiopsis nigra (Haw.) Rowley (2013a: 5)}

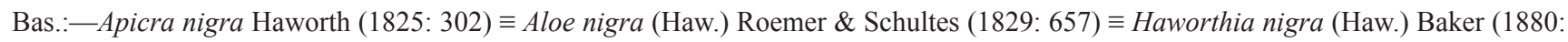
203) $\equiv$ Haworthia venosa subsp. nigra (Haw.) Halda (1997: 39) 三 Haworthia viscosa subsp. nigra (Haw.) Halda (1998: 44). Type (neotype designated by Breuer \& Metzing 1997: 10):-SOUTH AFRICA. Eastern Cape: Campherspoort [QDS: 3324AA], 1947, W.F. Barker 5099 (NBG0068251!). Type (lectotype designated by Breuer 2000: 600):- (icon) "Haworthia nigra" in (K).

Distribution:- It has a rather wide distribution and is found in the drier interior parts of the Western and Eastern Cape provinces of South Africa, from near Tsomo in the east to near Merweville in the west.

14a. var. nigra

= Haworthia schmidtiana Von Poellnitz (1929a: 23) EHaworthia nigra var. schmidtiana (Poelln.) Uitewaal (1948: 51)

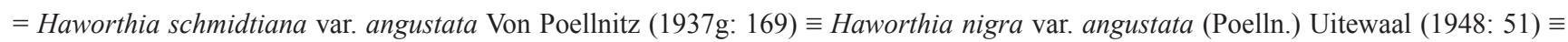
Haworthia nigra f. angustata (Poelln.) Pilbeam (1983: 101)

= Haworthia schmidtiana var. suberecta Von Poellnitz (1937g: 169) E Haworthia nigra var. suberecta (Poelln.) Uitewaal (1948: 51)

= Haworthia schmidtiana var. pusilla Von Poellnitz (1938b: 240) EHaworthia nigra var. pusilla (Poelln.) Uitewaal (1948: 51)

= Haworthia ryneveldii Von Poellnitz (1939b: 271)

Distribution:- The typical variety is found on the eastern end of the distribution range of the species where it is widespread in the interior parts of the Eastern Cape, South Africa. It is known as far east as the Tsomo River, and occurs in the Great Fish River catchment area and to the vicinity of Aberdeen in the west.

14b. var. diversifolia (Poelln.) Rowley (2013a: 4)

Bas.:-Haworthia diversifolia Von Poellnitz (1937a: 200) $\equiv$ Haworthia schmidtiana var. diversifolia (Poelln.) Von Poellnitz (1938b: 205) $\equiv$ Haworthia nigra var. diversifolia (Poelln.) Uitewaal (1948: 51). Type (lectotype designated by Breuer 1998: 135):- (icon) "Haworthia diversifolia" v.P. in (B). Type (epitype designated by Bayer 1999: 187):- SOUTH AFRICA. Eastern Cape: Willowmore, Kruidfontein [QDS: 3323AB], P.V. Bruyns in KG435/75 (NBG).

= Haworthia schmidtiana var. diversifolia f.nana Von Poellnitz (1938b: 240) 三 Haworthia nigra var. diversifolia f. nana (Poelln.) Uitewaal (1948: 51) E Haworthia nigra f. nana (Poelln.) Pilbeam (1983: 101)

Distribution:- This variety is known from the western limit of the distribution range of the species. It is found in the drier interior, particularly in the Great Karoo (Western Cape), but occurs from near Merweville (Western Cape) in the west, eastwards to just northeast of Willowmore (Eastern Cape), South Africa. 
14c. var. elongata (Poelln.) Rowley (2013a: 4)

Bas.:-Haworthia schmidtiana var. elongata Von Poellnitz (1938b: 240); Haworthia nigra var. elongata (Poelln.) Uitewaal (1948: 51). Type (lectotype designated by Breuer 1998: 237):- (icon) “Haworthia schmidtiana var. elongata” v.P. (B). Note: The neotypification by Bayer \& Manning (2012: 20) is superfluous as it is superceded by the lectotypification by Breuer (1998).

Distribution:- Not much is known about the distribution range of this taxon. The original collection is reported to have come from Bulkraal near Slagtersnek, Eastern Cape, South Africa.

\section{Haworthiopsis scabra (Haw.) Rowley (2013a: 5)}

Bas.:- Haworthia scabra Haworth (1819: 58). Type (lectotype designated by Scott 1980: 274):- (icon) (K), later published in Scott (1980: 274).

Distribution:- It is predominately found in the Little Karoo, from near Ladismith (Western Cape) in the west to near Willowmore (Eastern Cape) in the east, and extending further east to near Joubertina (Eastern Cape), South Africa. It is also found to the south, across the Outeniqua and Tsitsikamma mountains near Plettenberg Bay, Western Cape, South Africa.

\section{5a. var. scabra}

= Haworthia tuberculata Von Poellnitz (1931: 219) 三 Haworthia scabra var. tuberculata (Poelln.) Halda (1997: 35) 三 Haworthia scabra var. tuberculata (Poelln.) Hayashi (2000: 13)

= Haworthia tuberculata var. acuminata Von Poellnitz (1938b: 218)

= Haworthia tuberculata var. sublaevis Von Poellnitz (1938b: 219)

= Haworthia tuberculata var. subexpansa Von Poellnitz (1938d: 56)

= Haworthia tuberculata var. angustata Von Poellnitz (1940b: 27)

= Haworthia scabra var. johanii Hayashi (2001: 16) E Haworthia johanii (M.Hayashi) Breuer (2010: 7)

Distribution:- It is predominantly found in the Little Karoo, generally inland across the coastal mountains, known from near Ladismith (Western Cape) in the west, and eastwards to near Joubertina (Eastern Cape), South Africa.

15b. var. lateganiae (Poelln.) Rowley (2013a: 5)

Bas.:-Haworthia lateganiae Von Poellnitz (1937c: 103) 三 Haworthia starkiana var. lateganiae (Poelln.) Bayer (1976: 128) 三 Haworthia scabra var. lateganiae (Poelln.) Bayer (1999: 195). Type (lectotype designated by Breuer \& Metzing 1997: 12):- (icon) in (B), later published in Von Poellnitz (1937c: 103).

Distribution:- It has a limited distribution near Grootkruis in the area northeast of Oudtshoorn, Western Cape, South Africa.

15c. var. morrisiae (Poelln.) Rowley (2013a: 5)

Bas.:-Haworthia morrisiae Von Poellnitz (1937b: 132) 三 Haworthia scabra var. morrisiae (Poelln.) Bayer (1976: 137). Type (lectotype designated by Breuer \& Metzing 1997: 12):-(icon) Haworthia morrisiae "v.P. VI. 1937" in (B) later published in Von Poellnitz (1937b: 132).

Distribution:- It is known from the area around the Cango Caves and Koos Raubenheimer Dam to the north of Oudtshoorn, Western Cape, South Africa.

15d. var. smitii (Poelln.) Gildenh. \& Klopper comb. et stat. nov.

Bas.:- Haworthia smitii Von Poellnitz (1938e: 186) 三 Haworthia pumila var. smitii (Poelln.) Halda (1997: 36). Type (lectotype designated by Breuer 1998: 245):- (icon) “Haworthia smitii v.P.” (B) later published in Poellnitz (1938e: 187). 
Distribution:-Despite being well represented in collections, not much is known about the distribution of this variety, but it is reported to have come from the conglomerate hills to the east of Oudtshoorn, Western Cape, South Africa.

15e. var. starkiana (Poelln.) Rowley (2013a: 5)

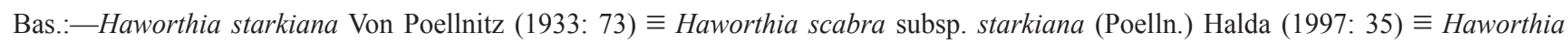
scabra var. starkiana (Poelln.) Bayer (1999: 197). Type (lectotype designated by Breuer \& Metzing 1997: 12):-(icon) "Haworthia starkiana v.P." (B).

Distribution:- It has a limited distribution in the area of Schoemanspoort, north of Oudtshoorn, Western Cape, South Africa.

16. Haworthiopsis viscosa (L.) Gildenh. \& Klopper comb. nov.

Bas.:-Aloe viscosa Linnaeus (1753: 332) 三 Haworthia viscosa (L.) Haworth (1812: 90) 三 Tulista viscosa (L.) Rowley (2013b: 6) [“Haworthiopsis viscosa (L.) Rowley” (2013a: 5) nom. inval. (Art. 36.2, McNeill et al., 2012); “Tulista viscosa (L.) Rowley” (2013a: 6) nom. inval. (Art. 36.2, McNeill et al., 2012)]. Type (lectotype designated by Scott 1981b: 98):- (icon) in Commelijn (1703: t.31). Type (epitype designated by Breuer \& Metzing 1997: 13):- SOUTH AFRICA. Western Cape: Blackburn Valley, Calitzdorp [QDS: 3321DA], 1947, W.F. Barker 5073 (NBG0068204!).

Distribution:- It is the third most widespread species after H. tessellata and H. nigra and occurs widely in the Western and Eastern Cape, South Africa.

16a. var. viscosa

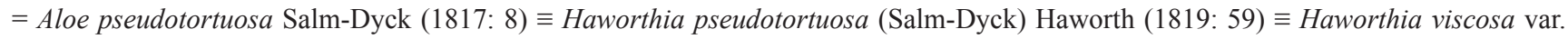
pseudotortuosa (Salm-Dyck) Baker (1880: 200); Haworthia viscosa f. pseudotortuosa (Salm-Dyck) Pilbeam (1983: 141)

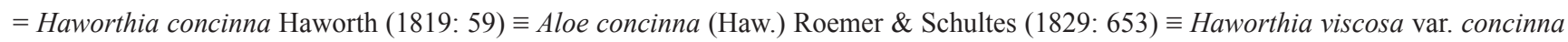
(Haw.) Baker (1880: 200)

= Haworthia asperiuscula Haworth (1819: 60) 三 Aloe asperiuscula (Haw.) Roemer \& Schultes (1829: 653) 三 Haworthia viscosa f. asperiuscula (Haw.) Pilbeam (1983: 141)

= Haworthia cordifolia Haworth (1819: 59) 三Aloe cordifolia (Haw.) Roemer \& Schultes (1829: 653)

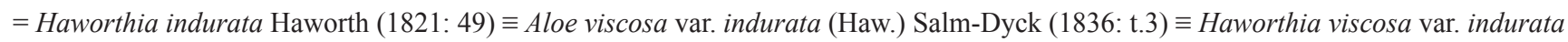
(Haw.) Baker (1880: 200)

= Haworthia viscosa var. major Haworth (1821: 49)

= Haworthia viscosa var. minor Haworth (1821: 49)

= Haworthia viscosa var. parvifolia Haworth (1821: 49)

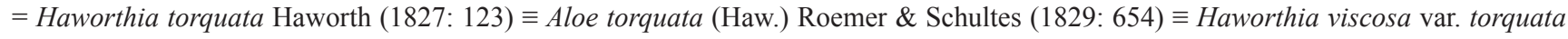
(Haw.) Baker (1880: 201); Haworthia viscosa f. torquata (Haw.) Pilbeam (1983: 141)]

= Aloe subtortuosa Roemer \& Schultes (1829: 654)

= Haworthia viscosa var. subobtusa Von Poellnitz (1938b: 240) EHaworthia viscosa f. subobtusa (Poelln.) Pilbeam (1983: 141)

= Haworthia viscosa var. caespitosa Von Poellnitz (1938b: 240)

= Haworthia beanii Smith (1944b: 137); 三 Haworthia viscosa f. beanii (G.G.Sm.) Pilbeam (1983: 141)

= Haworthia beanii var. minor Smith (1944b: 138)

= Haworthia viscosa var. cougaensis Smith (1945: 65)

= Haworthia viscosa var. viridissima Smith (1945: 67)

= Haworthia asperiuscula var. subintegra Smith (1945: 68)

= Haworthia asperiuscula var. patagiata Smith (1946: 11)

= Haworthia viscosa var. quaggaensis Smith (1948: 46)

= Haworthia viscosa subsp. derekii-clarkii Halda (1998: 43) as 'dereki-clarki'

Distribution:- It is found widespread in the Little Karoo especially around Calitzdorp and Oudtshoorn (Western Cape), as well as to the northwest near Laingsburg (Western Cape) and into the Eastern Cape, South Africa. 
16b. var. variabilis (Breuer) Gildenh. \& Klopper comb. nov.

Bas.:-Haworthia viscosa var. variabilis Breuer (2003: 61) 三 Haworthia variabilis (Breuer) Breuer (2010: 8) 三 Haworthiopsis variabilis (Breuer) Zonneveld (2014: 949). Type:-SOUTH AFRICA. Eastern Cape: Nordwestlich Joubertina [QDS: 3324CD], DMC 8979 in IB 7193 (holotype TUAT-RIEB)

Distribution:- It is found in a limited area north of Kareedouw in the Kouga Mountains, Eastern Cape, South Africa.

G. Haworthiopsis sect. Virescentes (Baker) Gildenh. \& Klopper comb. nov.

Bas.:- Haworthia sect. Virescentes Baker (1880: 199). Type (lectotype designated here):- Haworthiopsis sordida (Haw.) Rowley (2013a: 5) [=Haworthia sordida Haworth (1821: 51)].

17. Haworthiopsis bruynsii (M.B.Bayer) Rowley (2013a: 4)

Bas.:-Haworthia bruynsii Bayer (1981: 789) = Haworthia retusa subsp. emelyae var. bruynsii (M.B.Bayer) Halda (1997: 49). Type:SOUTH AFRICA. Eastern Cape: 20 km south east of Steytlerville [QDS: 3324BC], G.J. Rossouw 456 (holotype NBG0132381!).

Distribution:- It is found in a small area to the southeast of Steytlerville, an arid part of the Eastern Cape, South Africa.

\section{Haworthiopsis sordida (Haw.) Rowley (2013a: 4)}

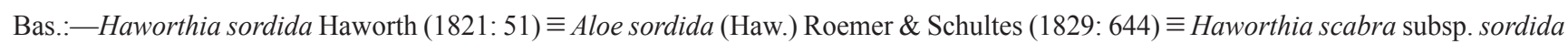
(Haw.) Halda (1997: 34). Type (neotype designated by Scott 1985: 7):- (icon) in Salm-Dyck (1836: Aloe t. 1 [sect 7: 2]).

Distribution:- It is known only from a narrow band in the Eastern Cape, South Africa, stretching from near Addo in the east to near Steytlerville in the west.

18a. var. sordida

= Haworthia agavoides Zantner \& Von Poellnitz (1938: 232) E Haworthia sordida var. agavoides (Zantner \& Poelln.) Smith (1950a: 2)

Distribution:- The typical variety is most widespread and is found across the range for the species from Kirkwood, Addo and Uitenhage in the east to near Kleinpoort in the west, Eastern Cape, South Africa.

18b. var. lavranii (C.L.Scott) Rowley (2013a: 4)

Bas.:-Haworthia sordida Haw. var. lavranii Scott (1981c: 124) 三 Haworthia scabra subsp. sordida var. lavranii (C.L.Scott) Halda (1997: 35) 三 Haworthia lavranii (C.L.Scott) Breuer (2010: 7). Type:-SOUTH AFRICA. Eastern Cape: Perdehoek, 36 km southeast of Steytlerville [QDS: 3324AC], A. Hechter s.n. (holotype PRE0616285!).

Distribution:- It is only known from a few populations near Steytlerville, Eastern Cape, South Africa.

\section{Haworthiopsis hybrids}

Even though Haworthiopsis species often co-occur with, or are found in close proximity to, species of Haworthia and Tulista, intergeneric hybrids are extremely rare (Rowley 2014: 21). Occasionally two species of Haworthiopsis are found in close proximity, usually $H$. viscosa with one of the other species, and this co-occurrence occasionally results in the presence of natural hybrids. Hybrids are known between $H$. viscosa and the following taxa: $H$. fasciata, H. glauca, H. longiana and H. scabra, some of which that are fertile. Bayer (1999) has also reported a natural hybrid between $H$. sordida and $H$. woolleyi. 


\section{New combinations in Haworthiopsis for hybrids formerly described as species of Haworthia}

There are several dubious taxa that have been previously described, some of which are obvious hybrids that have been given nothospecific epithets. In many cases it is uncertain if the hybrids were of horticultural origin or were introduced from the wild.

1. Haworthiopsis $\times$ broteriana (Resende) Gildenh. \& Klopper comb. nov.

Bas.:- Haworthia broteriana Resende (1941: 159) 三 Haworthia sampaiana f. broteriana (Resende) Resende \& Pinto Lopes (1946: 178). Type (neotype designated by Breuer 1998: 111):-Photographic illustration in Resende (1943: 16, fig.4a).

Notes:- This hybrid is of horticultural origin. The plant was cultivated and found at the botanical garden in Lisbon, Portugal. It is named in honour of the Portuguese botanist, Felix de Silva Avellar Brotero (1744-1828).

2. Haworthiopsis $\times$ cassytha (Baker) Gildenh. \& Klopper comb. nov.

Bas.:-Haworthia cassytha Baker (1896: 337). Type:- Not designated.

Notes:- - It is known that plant material was received from plant grower, Gebhart Ludwig Carl Pfersdorff (1815-1898) in 1875 and was cultivated at Kew (Breuer 2000). This plant is often considered synonymous with $H$. viscosa, but is a hybrid of this species.

3. Haworthiopsis $\times$ curta (Haw.) Gildenh. \& Klopper comb. nov.

Bas.:- Haworthia curta Haworth (1819: 60) $\equiv$ Aloe curta (Haw.) Sprengel (1825: 69) $\equiv$ Haworthia tortuosa var. curta (Haw.) Baker (1896: 336). Type:- Not designated.

Notes:- This hybrid has been cultivated at the Chelsea Physic Garden (England) since before 1817, and appears to be one of many hybrid plants that have been described. It was said to be much smaller (Haworth 1819) than H. ×tortuosa (Haworth 1804: 7) Gildenhuys \& Klopper comb. nov.

4. Haworthiopsis $\times$ expansa (Haw.) Gildenh. \& Klopper comb. nov.

Bas.:- Aloe expansa Haworth (1804: 8) 三 Haworthia expansa (Haw.) Haworth (1812: 91) 三 Aloe rigida var. expansa (Haw.) Salm Dyck (1836: t.3) 三 Haworthia rigida var. expansa (Haw.) Baker (1880: 203). Type (neotype designated by Breuer 1998: 139):-Illustration in Salm-Dyck (1836: Aloe t.9).

Notes:-This plant has been compared (Haworth 1812) with H. ×rigida (Lamarck 1783: 89) Gildenhuys \& Klopper comb. nov. in the past and is also of hybrid origin. It may be a synonym of $\mathrm{H}$. $\times$ rigida.

5. Haworthiopsis $\times$ hybrida (Salm-Dyck) Gildenh. \& Klopper comb. nov.

Bas.:-Aloe hybrida Salm-Dyck (1817: 7) 三 Haworthia hybrida (Salm-Dyck) Haworth (1821: 51). Type (neotype designated by Breuer 1998: 158):- Illustration in Salm-Dyck (1837: Aloe t.7).

Notes:-Originally described as Aloe hybrida Salm-Dyck from plants grown in the Botanical Garden of Vienna (Austria), and it was only later introduced to Kew.

6. Haworthiopsis $\times$ kewensis (Poelln.) Gildenh. \& Klopper comb. nov.

Bas.:- Haworthia kewensis Von Poellnitz (1940c: 57). Type (neotype designated by Breuer 1998: 163):-Photographic illustration in Resende (1943: 76, fig.30e).

Notes:- The type plant with the name "Haworthia peacockii" was received by Von Poellnitz from Kew in 1937, and was later described as Haworthia kewensis. 


\section{Haworthiopsis $\times$ lisbonensis (Resende) Gildenh. \& Klopper comb. nov.}

Bas.:-Haworthia lisbonensis Resende (1946: 175). Type (lectotype designated by Breuer 1998: 170):-Photographic illustration in Resende (1946: 179, fig.1).

Notes:-This plant was brought to the attention of Resende by a gardener, Mr. Gomes-Amarai at the Lisbon Botanical Garden in Portugal.

8. Haworthiopsis ×major (Salm-Dyck) Gildenh. \& Klopper comb. et. stat. nov.

Bas.:-Aloe tortuosa var. major Salm-Dyck (1837: t.6) 三 Haworthia tortuosa var. major (Salm-Dyck) Baker (1896: 336). Type (lectotype designated by Breuer 1998: 259): Photographic illustration in Salm-Dyck (1837: Aloe t.6).

Notes:- Initially described as a variety of Aloe tortuosa Salm-Dyck (1837). In later years Aloe pseudorigida SalmDyck (1817: 9), Haworthia curta Haworth (1819: 60) and Haworthia tortella Haworth (1819: 61) were transferred to Haworthia tortuosa (Haw.) Haw. as varieties thereof. All of these and H. tortuosa var. major (Salm-Dyck) Baker are of hybrid origin. Since each description was based on individual clones, and it is unlikely that wild populations exist or existed, it does not seem sensible to have hybrids classified as varieties of another hybrid. All of these are here treated as separate hybrid taxa at species level.

\section{Haworthia ×pseudorigida (Salm-Dyck) Gildenh. \& Klopper comb. nov.}

Bas.:-Aloe pseudorigida Salm-Dyck (1817: 9) $\equiv$ Apicra pseudorigida (Salm-Dyck) Haworth (1819: 62) $\equiv$ Haworthia tortuosa var. pseudorigida (Salm-Dyck) Berger (1908: 79). Type:-SOUTH AFRICA. Cape: Origin unknown. Not preserved.

Notes:-A hybrid of $H$. viscosa.

10. Haworthiopsis $\times$ resendeana (Poelln.) Gildenh. \& Klopper comb. nov.

Bas.:- Haworthia resendeana Von Poellnitz (1938f: 225). Type (lectotype designated by Breuer 1998: 225):-Photographic illustration "Haworthia Resendeana v.P." in (B); later published in Von Poellnitz (1938f: 226).

Notes:- This horticultural hybrid was named in honour of the Portuguese botanist, Flávio P. de Resende (19071967).

11. Haworthiopsis $\times$ revendettii (Uitewaal) Gildenh. \& Klopper comb. nov.

Bas.:- Haworthia revendettii Uitewaal (1940b: 44). Type (lectotype designated by Breuer 1998: 230):-Photographic illustration in Uitewaal (1940b: 44).

Notes:- This plant had been known as "Haworthia revendettii" for several years in cultivation prior to its official description. Even though the origin and the person after whom the plant was named were unknown, Uitewaal retained the name and described it officially in 1940.

12. Haworthiopsis $\times$ rigida (Lam.) Gildenh. \& Klopper comb. nov.

Bas.:- Aloe cylindrica var. rigida Lamarck (1783: 89) $\equiv$ Aloe rigida (Lam.) de Candolle (1799: 62) $\equiv$ Apicra rigida (Lam.) Willdenow (1811: 272) 三 Haworthia rigida (Lam.) Haworth (1821: 49). Type (neotype designated by Breuer 1998: 125):-Illustration in SalmDyck (1836: Aloe t.21).

Notes:- This plant appears to be a hybrid with H. viscosa. 


\section{Haworthiopsis $\times$ rubrobrunnea (Poelln.) Gildenh. \& Klopper comb. nov.}

Bas.:- Haworthia rubrobrunnea Von Poellnitz (1940c: 57). Type (neotype designated by Breuer 1998: 231):-Photographic illustration in Resende (1943: 91, fig.35c).

Notes:-Von Poellnitz received the plant labelled as "Haworthia no. 3" from Kew without any locality. This is a hybrid of unknown parentage but morphologically clearly belongs in Haworthiopsis. The name refers to the reddish brown leaf colouration.

14. Haworthiopsis $\times$ sampaiana (Resende) Gildenh. \& Klopper comb. nov.

Bas.:- Haworthia coarctata var. sampaiana Resende (1938: 177) 三 Haworthia sampaiana (Resende) Resende (1940: 192). Type (neotype designated by Breuer 1998: 120):- Photographic illustration in Resende (1943: 73, fig.28a).

Notes:-This plant has been growing in the Hamburg Botanic Garden (Germany) since as early as 1905. It was named in honour of the Portuguese botanist, Gonçalo António da Silva Ferreira Sampaio (1865-1937).

\section{Haworthiopsis $\times$ subrigida (Roem. \& Schult.) Gildenh. \& Klopper comb. nov.}

Bas.:-Aloe subrigida Roemer \& Schultes (1829: 654) 三 Haworthia subrigida (Roem. \& Schult.) Baker (1880: 201). Type (neotype designated by Breuer 2000: 759):-Illustration in Salm-Dyck (1836: Aloe t.7).

Notes:-This species was said to be intermediate between $H$. $\times$ tortuosa and $H . \times$ rigida and is of hybrid origin (Baker 1896). This plant was introduced to cultivation at Kew by Salm-Dyck.

16. Haworthiopsis $\times$ tauteae (Archibald) Gildenh. \& Klopper comb. nov.

Bas.:- Haworthia tauteae Archibald (1946: 992, as 'tautae'). Type:-SOUTH AFRICA. Western Cape Province: George district [QDS: 3322-CD], Taute s.n. (holotype, GRA).

Notes:-This plant was named in honour for Mrs. Taute who collected it in 1943. It is a hybrid between $H$. viscosa and H. scabra, and is reported by Bayer (1999) to have come from Kleinspoort, east of Oudtshoorn, South Africa.

17. Haworthiopsis $\times$ tortella (Haw.) Gildenh. \& Klopper comb. nov.

Bas.:- Haworthia tortella Haworth (1819: 61) 三 Haworthia tortuosa var. tortella (Haw.) Baker (1896: 336). Type:-Not designated.

Notes:-This hybrid was once included as a variety of $H . \times$ tortuosa.

18. Haworthiopsis $\times$ tortuosa (Haworth) Gildenh. \& Klopper comb. nov.

Bas.:- Aloe tortuosa Haworth (1804: 7) 三 Apicra tortuosa (Haw.) Willdenow (1811: 274) 三 Haworthia tortuosa (Haw.) Haworth (1812: 90). Type (neotype designated by Breuer 1998: 259):-Illustration in Salm-Dyck (1837: Aloe t.5).

Notes:-A widely cultivated hybrid of $H$. viscosa.

\section{Acknowledgements}

A great thank you and words of appreciation to the following people: Prof. Braam (A. E.) van Wyk (University of Pretoria) for helpful advice and assisting with the preparation of the manuscript; Brian Kemble (Ruth Bancroft Botanic Garden) for his dedication and patience with the editing of the draft manuscript; Giuseppe Orlando for the Latin diagnosis for the new section; Prof. John McNeill (Royal Botanic Garden, Edinburg) for valuable nomenclatural advice; two anonymous reviewers and the editor for suggesting improvements to the manuscript. 


\section{References}

Angiosperm Phylogeny Group (2009) An update of the Angiosperm Phylogeny Group classification for the orders and families of flowering plants: APG III. Botanical Journal of the Linnean Society 161: 105-121.

http://dx.doi.org/10.1111/j.1095-8339.2009.00996.x

Angiosperm Phylogeny Group (2016) An update of the Angiosperm Phylogeny Group classification for the orders and families of flowering plants: APG IV. Botanical Journal of the Linnean Society 181: 1-20.

http://dx.doi.org/10.1111/boj.12385

Archibald, E.E.A. (1946) Haworthia tautae. The Flowering Plants of Africa 25: t. 992.

Baker, J.G. (1880) Synopsis of the Aloineae and Yuccoideae. Journal of the Linnean Society, Botany 18 (109): $197-216$. http://dx.doi.org/10.1111/j.1095-8339.1880.tb00663.x

Baker, J.G. (1896) Haworthia Duval. In: Thistelton-Dyer, W.T. (Ed.) Flora Capensis 6. L. Reeve \& Co., London, pp. $333-355$.

Barker, W.F. (1937) Plantae novae Africanae, ser. VIII. Journal of South African Botany 3: 92-95.

Bayer, M.B. (1962) A new variety of Haworthia limifolia from Natal. Journal of South African Botany 28: 215-217.

Bayer, M.B. (1971) Changes in the genus Haworthia. Cactus and Succulent Journal (US) 43: 157-162.

Bayer, M.B. (1973) Natural variation and species delimitation in Haworthia Duval, Part 3: Haworthia reinwardtii and Haworthia coarctata Haw. National Cactus \& Succulent Journal (GB) 28: 80-86.

Bayer, M.B. (1976) Haworthia Handbook. National Botanic Gardens of South Africa, Kirstenbosch, 184 pp.

Bayer, M.B. (1982) The new Haworthia Handbook. National Botanic Gardens of South Africa, Kirstenbosch, 124 pp.

Bayer, M.B. (1999) Haworthia revisited: A revision of the genus. Umdaus Press, Pretoria, 188 pp.

Bayer, M.B. (2003) Whereto Haworthia limifolia? Aloe 40: 41-51.

Bayer, M.B. \& Manning, J.C. (2012) A rationalisation of names in Haworthia: a list of new combinations and new synonyms. Haworthia Update, Essays on Haworthia. Alsterworthia International 7: 30-40.

Berger, A. (1908) Liliaceae-Asphodeloideae-Aloineae. In: Engler, A. \& Prantl, K. (Eds.) Das Pflanzenreich IV. 38 (Heft 33 ). Engelmann, Leipzig, $347 \mathrm{pp}$.

Bolus, H.M.L. (1920) Novitates africanae: Apicra rubriflora L. Bolus (Liliaceae-Aloineae). Annals of the Bolus Herbarium 3: 1-14.

Breuer, I. \& Metzing, D. (1997) Types of names accepted in Haworthia (Aloaceae). Taxon 46: 3-14. http://dx.doi.org/10.2307/1224286

Breuer, I. (1998) The World of Haworthias 1. Ingo Breuer and Arbeitskreis fur Mammillarienfreunde e.V. (AfM), Niederzier and Homburg/ Saar, pp. 1-340.

Breuer, I. (2000) The World of Haworthias 2. Ingo Breuer and Arbeitskreis fur Mammillarienfreunde e.V. (AfM), Niederzier and Homburg/ Saar, pp. 341-859.

Breuer, I. (2003) Beschreibung einiger neuer Haworthia-Taxa. Avonia 21: 45-64.

Breuer, I. (2010) The genus Haworthia: Part 1. Alsterworthia International, Preston, UK 86 pp.

Brown, N.E. (1876) New garden plants. Gardener's Chronicle 6: 130.

Commelijn, C. (1703) Praeludia Botanica. Fredericum Haringh, Amsterdam, 86 pp. +33 figs.

Daru, B.H., Manning, J.C., Boatwright, J.S., Maurin, O., Maclean, N., Schaefer, H., Kuzmina, M. \& Van der Bank, M. (2013) Molecular and morphological analysis of subfamily Alooideae (Asphodelaceae) and the inclusion of Chortolirion in Aloe. Taxon 62: 62-76.

De Candolle, A.P. (1799) Plantarum Historia Succulentarum (Histoire des plante grasses) 2. A.J. Dugour et Durand, Paris, 104 pp., 52 plates.

Dinter, M.K. (1914) Neue und wenig bekannte Pflanzen Seutsch-Südwest-Afrikas. The Author, Okahandja, 63 pp.

Duval, H.A. (1809) Plantae Succulentae in Horto Alenconio. Gabon, Paris, 18 pp.

Farden, R.S. (1939) Haworthia attenuata and its varieties. The Cactus Journal (GB) 8: 34-38.

Grace, O.M., Klopper, R.R., Smith, G.F., Crouch, N.R., Figueiredo, E., Rønsted, N., Van Wyk, A.E. (2013) A revised generic classification for Aloe (Xanthorrhoeaceaea subfam. Asphodeloideae). Phytotaxa 76 (1): 7-14.

http://dx.doi.org/10.11646/phytotaxa.76.1.2

Halda, J.J. (1997) Synopsis of the genus Haworthia (Duval), with some nomenclatoric changes. Acta Musei Richnoviensis, Sect Nat. 4: $33-51$.

Halda, J.J. (1998) New descriptions. Acta Musei Richnoviensis, Sect Nat. 5: 40-47.

Haworth, A.H. (1804) A new arrangement of the genus Aloe. Transactions of the Linnaean Society of London 7: 1-28. http://dx.doi.org/10.1111/j.1096-3642.1804.tb00276.x

Haworth, A.H. (1812) Synopsis Plantarum Succulentarum. Taylor, London, 207 pp.

Haworth, A.H. (1819) Supplementum Plantarum Succulentarum. R. \& A. Taylor, London, 160 pp. 
Haworth, A.H. (1821) Revisiones Plantarum Succulentarum. R. \& A. Taylor, London, 207 pp.

http://dx.doi.org/10.5962/bhl.title.9670

Haworth, A.H. (1824) Decas Secunda Novarum Plantarum Novarum Succulentarum. Philosophical Magazine \& Journal 64: $298-302$.

Haworth, A.H. (1825) Decas Quinta Novarum Plantarum Succulentarum. Philosophical Magazine \& Journal 66: $279-283$.

Haworth, A.H. (1827) Decas Octava Plantarum Novarum Succulentarum. The Philosophical Magazine ser. 2 3: 120-123. http://dx.doi.org/10.1080/14786442708674240

Haworth, A.H. (1828) Decas Undecima Plantarum Novarum Succulentarum. The Philosophical Magazine ser.2 3: $183-188$. http://dx.doi.org/10.1080/14786442808674606

Hayashi, M. (2000) Comparisson of Haworthia species names. Haworthia Study 3: 5-13.

Hayashi, M. (2001) Some new species in Haworthia. Haworthiad 15: 16-20.

Hayashi, M. (2003) Haworthia schoemanii Hayashi sp. nov. Haworthia Study 9: 14.

Hjelmquist, H. (1943) Notiser fran Lunds Botaniska Trädgard V. Botaniska Notiser 1943: 243-246.

Jacquin, N.J. (1804) Plantarum Rariorum Horti Caesarei Schoenbrunnensis, Wien 4. C.F. Wappler, Vienna, 56 pp. + tab.100.

Klopper, R.R., Smith, G.F. \& Van Wyk, A.E. (2013). (2129) Proposal to conserve the family name Asphodelaceae (Spermatophyta:

Magnoliidae: Asparagales). Taxon 62: 402-403.

http://dx.doi.org/10.12705/622.17

Lamarck, J.B.A.P.M. de (1783) Encyclopédie Méthodique, Botanique 1. Panckoucke, Paris, 748 pp.

Linnaeus, C. (1753) Species Plantarum 1. Impensis Laurentii Salvii, Stockholm, 560 pp.

Manning, J.C. \& Smith, G.F. (2000) The genus Poellnitzia included in Astroloba. Bothalia 30: 53.

Manning, J.C., Boatwright, J.S., Daru, B.H., Maurin, O. \& Van der Bank, M. (2014) A molecular phylogeny and genetic classification of Asphodelaceae subfamily Alooideae: A final resolution of the prickly issue of polyphyly in the alooids? Systematic Botany 39: $55-74$.

http://dx.doi.org/10.1600/036364414X678044

Marloth, H.W.R. (1910) Some new South African succulents, part 2, Haworthia limifolia. Transactions of the Royal Society of South Africa 1: 409.

http://dx.doi.org/10.1080/00359191009520051

Marloth, H.W.R. (1910) Some new South African succulents, part 3, Haworthia granulata. Transactions of the Royal Society of South Africa 2: 39.

http://dx.doi.org/10.1080/00359191009519359

Marloth, H.W.R. \& Berger, A. (1906) Neue Aloineen und andere Sukkulenten. Notisblatt des Königlichen Botanischen Gartens und Museums zu Berlin 4(38): 247-248.

McNeill, J., Barrie, F.R., Buck, W.R., Demoulin, V., Greuter, W., Hawksworth, D.L., Herendeen, P.S., Knapp, S., Marhold, K., Prado, J., Prud'homme van Reine, W.F., Smith, G.F., Wiersema, J.H. \& Turland, N.J. (Eds.) (2012) International Code of Nomenclature for algae, fungi, and plants (Melbourne Code) adopted by the Eighteenth International Botanical Congress Melbourne, Australia, July 2011. Koeltz Scientific Books, Königstein. [Regnum Vegetabile 154], xxx + 208 pp.

Nyffeler, R. \& Eggli, U. (2010) An up-to-date familial and suprafamilial classification of succulent plants. Bradleya 28: 125-144.

Obermeyer, A.A. \& Hardy, D.S. (1967) Haworthia koelmaniorum sp. nov. Flowering plants of Africa 38: t.1502.

Pilbeam, J. (1983) Haworthia and Astroloba: A collector's guide. B.T. Batsford Ltd., London, 167 pp.

Rafinesque, C.S. (1840) Autikon Botanikon. Philadelphia, 200 pp.

Resende, F. (1938) Haworthia coarctata var. sampaiana var. nov. Feddes Repertorium Specierum Novarum Regni Vegetabilis 45: 177178.

http://dx.doi.org/10.1002/fedr.19380450806

Resende, F. (1940) Estudos Caryologicas nas Aloineae, 3. Apoloidea na seccao Tessellatae do genera e as actuais leis de prioridade em sistematica. Boletim da Sociedade Broteriana, Lissabon 14: 189-204.

Resende, F. (1941) Suculentas Africanas I: Tres especies novas de Haworthia Duval. Boletim da Sociedade Broteriana, Lissabon 15: $159-162$.

Resende, F. \& Von Poellnitz, K. (1942) Suculentas Africana II: Variedades novas de Haworthia tessellata Haw. Revista (da Sociedade) Broteriana, Serie Ciencias Naturales 11: 49-55.

Resende, F. (1943) Suculentas Africanas III: Contribuicao para o estude da morfolofia, da fisiologia da floracao e da genosistematica das Aloinae. Memoirias de la Sociedade Broteriana 2: 1-119.

Resende, F. (1946) Suculentas Africanas IV: Contribution to a better geno-sistematical knowledge of the Coarctatae section of the genus Haworthia Duval. Portugaliae Acta Biologica, Serie B 2: 177-196.

Resende, F. \& Pinto-Lopes, J. (1946) Cariologia de seccao Coarctatae Berger do genero Haworthia Duval. Agronomia Lusitana 6: $129-212$. 
Resende, F. \& Pinto-Lopes, J. (1948) Caryological studies in the Aloinae V, Contribution to the knowledge of the genus Haworthia. Portugaliae Acta Biologica, Serie A 2: 175-190.

Roemer, J.J. \& Schultes, J.A. (1829) Systema Vegetabilum, Stuttgart 7: 631-715.

Rowley, G.D. (1985) The Haworthia drawings of John Thomas Bates. Succulent Plant Trust, Buckhurst Hill, 58 pp.

Rowley, G.D. (2013a) Generic concepts in Alooideae - Part 3: The phylogenetic story. Alsterworthia International Special Issue 10: 3-6. [Published 9 April 2013]

Rowley, G.D. (2013b) Generic concepts in Alooideae - Part 3 Amended: The phylogenetic story. Alsterworthia International Special Issue 10: $3-6$.

Rowley, G.D. (2014) Intergeneric hybrids in the Asphodelaceae subfamily Alooideae - 2014 update. Alterworthia International 4: 1623.

Rowley, G.D. (2015) Apologia, Corrigendum. Alsterworthia International 15: 2.

Salm-Reifferscheid-Dyck, Prince J.M.F.A.H.I. von (1817) Catalogue raisoné des Espèces d'Aloes. Düsseldorf, 72 pp.

Salm-Reifferscheid-Dyck, Prince J.M.F.A.H.I. von (1821) Obsevationes Botanicae in Horto Dyckensi. Köln, 46 pp.

Salm-Reifferscheid-Dyck, Prince J.M.F.A.H.I. von (1829) Index Plantarum Succulentarum in Horto Dyckensi Cultarum. Aquisgrani, 71 pp.

Salm-Reifferscheid-Dyck, Prince J.M.F.A.H.I. von (1834) Hortus Dyckensis. Arnz \& Co., Düsseldorf, 376 pp.

Salm-Reifferscheid-Dyck, Prince J.M.F.A.H.I. von (1836) Monographia generum Aloes et Mesembryanthemi 1. Henry \& Cohen, Düsseldorf \& Bonn, 60 tab.

Salm-Reifferscheid-Dyck, Prince J.M.F.A.H.I. von (1837) Monographia generum Aloes et Mesembryanthemi 2. Henry \& Cohen, Düsseldorf \& Bonn, 58 tab.

Salm-Reifferscheid-Dyck, Prince J.M.F.A.H.I. von (1840) Monographia generum Aloes et Mesembryanthemi 3. Henry \& Cohen, Düsseldorf \& Bonn, 57 tab.

Salm-Reifferscheid-Dyck, Prince J.M.F.A.H.I. von (1849) Monographia generum Aloes et Mesembryanthemi 5. Henry \& Cohen, Düsseldorf \& Bonn, 54 tab.

Scott, C.L. (1978) The reinstatement of Haworthia species. Cactus \& Succulent Journal (US) 50: 74-77.

Scott, C.L. (1980) Diversity in the species Haworthia scabra Haw. and H. tuberculata von Poelln. Cactus and Succulent Journal (U.S.) 52: 274-276.

Scott, C.L. (1981a) Haworthia reinwardtii Haw. National Cactus \& Succulent Journal (GB) 36: 36-40.

Scott, C.L. (1981b) Haworthia viscosa (L.) Duval. National Cactus \& Succulent Journal (GB) 36: 98-100.

Scott, C.L. (1981c) A new variety of Haworthia Duval. Cactus \& Succulent Journal (US) 53: 124-126.

Scott, C.L. (1984) A new species of Haworthia (Liliaceae-Aloineae) sect. Retusae. Cactus \& Succuletn Journal (US) 56: 69-70.

Scott, C.L. (1985) The genus Haworthia (Liliaceae). A taxonomic revision. Aloe Books, Johannesburg, 150 pp.

Smith, G.F. \& Crouch, N.R. (2001) Haworthia limifolia var. arcana (Asphodelaceae: Alooideae): a new variety from eastern South Africa. Bradleya 19: 117-120.

Smith, G.F. \& Greyling, H.P. (1990) The neotypification of Aloe glabrata, basionym of Haworthia glabrata (Asphodelaceae: Alooideae). Taxon 39: 331-332. http://dx.doi.org/10.2307/1223064

Smith, G.G. (1943) Some new species and varieties in the genus Haworthia I. Journal of South African Botany 9: 93-107.

Smith, G.G. (1944a) Some new species and varieties in the genus Haworthia II. Journal of South African Botany 10: 11-24.

Smith, G.G. (1944b) Some new species and varieties in the genus Haworthia III. Journal of South African Botany 10: 137-143.

Smith, G.G. (1945) Some new species and varieties in the genus Haworthia IV. Journal of South African Botany 11: 65-78.

Smith, G.G. (1946) Some new species and varieties in the genus Haworthia V. Journal of South African Botany 12: 1-14.

Smith, G.G. (1948) Some new species and varieties in the genus Haworthia VI. Journal of South African Botany 14: 41-54.

Smith, G.G. (1950a) Some transfers in the genus Haworthia VII. Journal of South African Botany 16: 1-2.

Smith, G.G. (1950b) Some new species and varieties in the genus Haworthia. Journal of South African Botany 16: 3-13.

Thiers, B. (2015) Index herbariorum, a global directory of public herbaria and associated staff. New York Botanical Garden's Virtual Herbarium. Available from: http://sweetgum.nybg.org/science/ih/ (accessed 28 April 2016)

Treutlein, J., Smith, G.F., Van Wyk, B-E. \& Wink, M. (2003) Phylogenetic relationships in Asphodelaceae (subfamily Alooideae) inferred from chloroplast DNA sequences (rbcL, matK) and from genomic fingerprinting (ISSR). Taxon 52: 193-207. http://dx.doi.org/10.2307/3647389

Uitewaal, A.J.A. (1940a) Een nieuw geslacht der Aloineae. Succulenta 22: 61-64.

Uitewaal, A.J.A. (1940b) Twee bekende doch onbeschreven Haworthia's. Cactussen \& Vetplanten 6: 44.

Uitewaal, A.J.A. (1947a) A first attempt to subdivide the genus Haworthia, based on floral characters. Desert Plant Life 19: 133-138. Uitewaal, A.J.A. (1947b) Revision of the nomenclature of the genera Haworthia and Apicra. Succulenta 26: 51-54. 
Uitewaal, A.J.A. (1948) Revisie van de nomenclatuur van enkele Haworthia's (II). Succulenta 27: 49-52.

Van Woerden, C. (1940) Haworthia tesselata var. elongata. Succulenta 22: 37-40.

Verdoorn, I. (1941) Haworthia ubomboensis. The Flowering Plants of South Africa 21: t.818.

Viveiros, A. (1949) Karyological studies in the Aloineae VI: polyploidy, asynchronous chromonemata multiplication and general karyotype. Portugaliae Acta Biologica, Serie A 3: 200-229.

Von Poellnitz, K. (1929a) Afrikanische Sukkulenten. Feddes Repertorium Specierum Novarum Regni Vegetabilis 26: 20-24. http://dx.doi.org/10.1002/fedr.19290260104

Von Poellnitz, K. (1929b) Zur Kenntis der Gattungen Haworthia Duval. und Gasteria Duval. Feddes Repertorium Specierum Novarum Regni Vegetabilis 27: 132-143.

http://dx.doi.org/10.1002/fedr.4870270903

Von Poellnitz, K. (1931) Haworthia tuberculata v.P. spec.nov. Feddes Repertorium Specierum Novarum Regni Vegetabilis 29: 219. http://dx.doi.org/10.1002/fedr.19310291109

Von Poellnitz, K. (1933) Zur Kenntis der Gattung Haworthia Duval. und Gasteria Duval. Feddes Repertorium Specierum Novarum Regni Vegetabilis 31: 82-91. http://dx.doi.org/10.1002/fedr.19320310108

Von Poellnitz, K. (1937a) Zur Kenntnis der Gattung Haworthia Duval. Feddes Repertorium Specierum Novarum Regni Vegetabilis 41: $193-220$

http://dx.doi.org/10.1002/fedr.19370411402

Von Poellnitz, K. (1937b) Neue Formen der Gattung Haworthia Duval. Kakteenkunde 9: 132-135.

Von Poellnitz, K. (1937c) Six new species of Haworthia Duval. Desert Plant Life 9: 101-103.

Von Poellnitz, K. (1937d) Vier neue Haworthia-arten. Kakteenkunde 10: 152-154.

Von Poellnitz, K. (1937e) Haworthia attenuata Haw. Kakteen und andere Sukkulenten 1: 165-166.

Von Poellnitz, K. (1937f) Vier neue Haworthia-arten. Feddes Repertorium Specierum Novarum Regni Vegetabilis 42: $269-271$. http://dx.doi.org/10.1002/fedr.19370421607

Von Poellnitz, K. (1937g) Haworthia schmidtiana v.P. und verwandte Formen. Kakteen und andere Sukkulenten 1: 168-170.

Von Poellnitz, K. (1938a) Haworthia fasciata (Willd.) Haw. und verwandte Formen. Feddes Repertorium Specierum Novarum Regni Vegetabilis 43: 94-97.

http://dx.doi.org/10.1002/fedr.19380430109

Von Poellnitz, K. (1938b) Über Sektionen der Gattung Haworthia Duval. Feddes Repertorium Specierum Novarum Regni Vegetabilis 44: $200-241$.

http://dx.doi.org/10.1002/fedr.19380441105

Von Poellnitz, K. (1938c) Haworthia woolleyi V.Poelln. The Cactus Journal (G.B.) 7: 3.

Von Poellnitz, K. (1938d) Haworthia tuberculata V.Poelln. and its varieties. The Cactus Journal (G.B.) 7: 56-57.

Von Poellnitz, K. (1938e) A new species of the genus Haworthia Duval. Desert Plant Life 10: 186-187.

Von Poellnitz, K. (1938f) Three new or little known succulent plants. Desert Plant Life 10: 225-226.

Von Poellnitz, K. (1939a) Four new species of Haworthia Duval. Desert Plant Life 11: 192-195.

Von Poellnitz, K. (1939b) Zwei neue Haworthia-Arten. Feddes Repertorium Specierum Novarum Regni Vegetabilis 46: $271-272$.

Von Poellnitz, K. (1940a) Haworthia reinwardtii Haw. Beiträge zur Sukkulentenkunde und -pflege 2: 41-43.

Von Poellnitz, K. (1940b) Zur Kenntnis von Haworthia Duval. Feddes Repertorium Specierum Novarum Regni Vegetabilis 49: 23-30. http://dx.doi.org/10.1002/fedr.19400490106

Von Poellnitz, K. (1940c) Zwei neue Haworthia-Arten. Feddes Repertorium Specierum Novarum Regni Vegetabilis 49: 57-58. http://dx.doi.org/10.1002/fedr.19400490502

Willdenow, C.L. (1811) Bemerkungen über die Gattung Aloe. Magazin der Gesellschaft naturforschender Freunde de zu Berlin 5: 267274.

Zantner, A. \& Von Poellnitz, K. (1938) Eine neue Haworthia-Art. Feddes Repertorium Specierum Novarum Regni Vegetabilis $43: 232$. http://dx.doi.org/10.1002/fedr.19380431106

Zonneveld, B.J.M. (2014) Nuclear genome sizes of 343 accessions of wild collected Haworthia and Astroloba (Asphodelaceae, Alooideae), compared with the genome sizes of Chortolirion, Gasteria and 83 Aloe species. Plant Systematics and Evolution 301: 931-953. http://dx.doi.org/10.1007/s00606-014-1127-4 